\title{
Natural cybernetics and mathematical history: the principle of least choice in history
}

\author{
Vasil Penchev, vasildinev@gmail.com \\ Bulgarian Academy of Sciences: Institute of Philosophy and Sociology: \\ Dept. of Logic and Philosophy of Science
}

\begin{abstract}
The paper follows the track of a previous paper "Natural cybernetics of time" in relation to history in a research of the ways to be mathematized regardless of being a descriptive humanitarian science withal investigating unique events and thus rejecting any repeatability. The pathway of classical experimental science to be mathematized gradually and smoothly by more and more relevant mathematical models seems to be inapplicable. Anyway quantum mechanics suggests another pathway for mathematization; considering the historical reality as dual or "complimentary" to its model. The historical reality by itself can be seen as mathematical if one considers it in Hegel's manner as a specific interpretation of the totality being in a permanent selfmovement due to being just the totality, i.e. by means of the "speculative dialectics" of history, however realized as a theory both mathematical and empirical and thus falsifiable as by logical contradictions within itself as emprical discrepancies to facts. Not less, a Husserlian kind of "historical phenomenology" is possible along with Hegel's historical dialectics sharing the postulate of the totality (and thus, that of transcendentalism). One would be to suggest the transcendental counterpart: an "eternal", i.e. atemporal and aspatial history to the usual, descriptive temporal history, and equating the real course of history as with its alternative, actually happened branches of the regions of the world as with only imaginable, counterfactual histories. That universal and transcendental history is properly mathematical by itself, even in a neoPythagorean model. It is only represented on the temporal screen of the standard historiography as a discrete series of unique events. An analogy to the readings of the apparatus in quantum mechanics can be useful. Even more, that analogy is considered rigorously and logically as implied by the mathematical transcendental history and sharing with it the same quantity of information as an invariant to all possible alternative or counterfactual histories. One can involve the hypothetical external viewpoint to history (as if outside of history or from "God's viewpoint to it), to which all alternative or counterfactual histories can be granted as a class of equivalence sharing the same information (i.e. the number choices, but realized in different sequence or adding redundant ones in each branch) being similar and even mathematically isomorphic to Feynman trajectories in quantum mechanics. Particularly, a fundamental law of mathematical history, the law of least choice of the real historical pathway is deducible from the same approach. Its counterpart in physics is the well-known and confirmed law of least action as far as the quantity of action corresponds equivocally to the quantity of information or that of number elementary historical choices.
\end{abstract}

Key words: Gadamer, Hegel, Heidegger, Husserl, mathematical and historical dialectics, mathematical and historical hermeneutics, mathematical and historical phenomenology, information conservation, mathematical history, natural historical cybernetics, transcendental history, law (principle) of least choice 
I INTRODUCTION: WHY HISTORY TO BE MATHEMATIZED IS SO DIFFICULT

History, as it is, is a descriptive, humanitarian, interpretative science withal referring to unique, unrepeatable events chosen to be studied because of a certain ideological viewpoint though unarticulated as usual. So, history has troubles not only to be mathematized, but even to be an objective science. For example, the science of history developed in the state A can be quite different form that in the neighboring state B: for this, the A historians is sufficient to choose different events to be studied rather than those chosen by their B colleagues. This means that history is an interpretative science crucially depending on the choice of the scientists who write it.

However, it is furthermore a humanitarian and social science therefore influenced not less crucially by the values, interpretations, minds (speaking more generally) of the actors "creating history" or by the public opinion and attitudes, official and generally accepted norms and preferences as well as many other subjective factors, agents, media, etc. (e.g. quite different in the state $\mathrm{A}$ in comparison to the state B).

Even more, it is a descriptive science "prohibiting the conditional mood", in which as any experiment as any prognosis to the future would be to be thought. This implies further that any mathematical models in that "unconditional history" are impossible. Indeed, there exist (1) comparative history studying similarity and differences in different countries or civilizations, (2) alternative history researching a certain history from another viewpoint (e.g. that of other country or civilization or postulating another "reference frame" to the own history), or even (3) counterfactual history inherently written in the forbidden "conditional mood". However, all of them rely on a much more unstable base than that of the standard history itself too doubtless as a mathematizable or even only objective science. This is the reason for the comparative, alternative and especially counterfactual history to be enumerated often among the area of literary fiction rather that to that of science.

To be, anyway, an objective science, history restricts itself only in a standard, preferably generally accepted tuple of what "historical facts" are and are to be described in a uniform manner: that is history as a science need be "factual historiography" relating only to the facts of the past rather than a speculative hermeneutics whether ideological or philosophical. So and rather paradoxically, history opposes particularly its objectivity to its eventual mathematization, an opposition unique for history among all sciences.

Still one "anti-mathematical" detail in history: it postulates (and this is justified) for all historical events to be unique and even: "the less unique, the less historical". On the contrary, any mathematical model applied in science follows and implies repeatability and predictability, or in the terms of the history itself, no historicity in the final analysis.

Summarizing, history seems to be one of the least suitable science to be mathematized.

As a paradigm of mathematization, physics (and more exactly quantum mechanics and quantum information) will be utilized further in the paper. Thus, a comparison of history to physics in relation to the premises for mathematization makes sense:

History is descriptive, and physics is grounded on a few principles, on an exhaustive list of them withal confirmed experimentally without any exceptions. The historical research is linked as a discourse, a narrative story unlike the physical one being a deduction to confirmable (respectively refutable) corollaries therefore directly interpretable in terms of experimental science. 
History is an interpretative science consequently including interpretations of the researcher in the studied facts inseparably and indistinguishably. Thus, history turns out to be unique involving "two times": once for the historical events are unique in definition, twice for the individuality of the researcher nuancing the facts by it.

On the contrary, physics considers any fact claiming to be physical as repeatable and absolutely independent of any human interpretation including that of the scientist who investigates. However, one should mean furthermore the troubles about the establishment of quantum mechanics referred also to the way and entity ("wave function", for example) able to be repeatable (since the single results of quantum measurements turning out to be fundamentally random $)^{1}$.

History is a humanitarian science, and thus based on human free will, and physics is a natural science postulating necessity, investigating natural laws, and building a form of cognition independent in the sense of invariance to human free will ${ }^{2}$.

\section{THE "NATURAL CYBERNETICS OF TIME"}

In the middle of the 20th century, Norbert Wiener suggested the idea of a new science, "cybernetics", a science studying the general laws of control and communication in biological and technical systems. It was related to social systems very soon, to any systems at all as "systemology" and it is the base of the contemporary computer science, neuroscience, cognitive science, etc.

Its revolutionary approach unifies the concepts of "system", "control", "communication", and "information", on the one hand, but neglects whether the systems are biological or social, or technical, or theoretical and abstract, or any others as well as whether they are the models of systems or the real systems themselves, on the other hand.

So, "system" becomes even a philosophical category, though implemented in a rigorously defined scientific notion, able to resolve the philosophical dualism of Modernity (e.g. "body" versus "mind" or "subject" versus "object") by mathematical isomorphism, i.e. as two different interpretations of the same, namely "system".

Thus, the concept of system turns out to be for science what the "totality" for philosophy is. For example, it generates an image of environment in the system and by itself, due only to the definitive property of being a system properly.

However, its revolution was restricted, not referring to physics, history, and not connecting to the research of the totality in philosophy, e.g. in Kant's transcendentalism or Hegel's dialectics, in Husserl's phenomenology or Heidegger's and Gadamer's hermeneutics. It utilized mathematics only as a tool (just as classical science did), but not considering it as a system to be investigated as such and thus, absolutely divided from the research of the foundations of mathematics undertaken by mathematics to ground its consistent completeness, i.e. considering itself as a system implicitly and intending to prove this.

${ }^{1}$ There exist even philosophical speculations reckoning the observer or experimenter (or their consciousness) of any quantum measurement as a "hidden variable" able to determine additionally and unambiguously the result.

${ }^{2}$ That relation of physics to free will is revised in a certain rigorous meaning established by the "free will theorems" (Conway, Kochen 2006; 2009) based on a few statements in quantum mechanics and special relativity. 
Meanwhile, quantum mechanics reformulated itself as an informational theory thoroughly in the end of the last century: the theory of quantum information. A concept of cybernetics, namely "information" was proclaimed to be natural, as "quantum information", and then heralded as the most general and omnipresent substance of the world while energy and matter can be defined restrictively physically, only to the "temporal screen of the being" (i.e. interpreting Heidegger's "Sein" and "Zeit" in a rigorous physical and mathematical manner in accordance to both transcendentalism and dialectics both physical and mathematical as in: Penchev 2020 October 20).

An immense area of research, including experimental, was open: that of quantum information and the phenomena of entanglement and resulting particularly into "dark matter" and "dark energy" absolutely unexplainable by the Standard model of the contemporary quantum physics identifying the temporal screen of the physical being with the physical being by itself: i.e. incapable to see what is "behind the temporal screen", where quantum information and phenomena of entanglement would be to be situated and appearing only as Plato's "shadows" projected on the "wall of the space-time cave".

So, time, on the "wall screen" of which all is well-ordered (even in a rigorous mathematical meaning), implies a physical and not less mathematical cybernetics, however at the cost of cancelling the option for studying anything out of the "space-time cave".

The mathematical counterpart of the projection on the "cave wall" is the well-known and elementarily provable equivalence of the well-ordering "theorem" and the axiom of choice therefore physically relating time (as the "quantity of well-ordering") to information (as the "quantity of choices".

Just that natural cybernetics of time is the correct viewpoint to history to be mathematized consequently researching the informational laws of mathematical history: a thesis advocated by the present paper as a main objectivity.

\section{THE TOTALITY: TIME AND INFORMATION}

The conjectured quantities of time and information can be tracked back to the totality once it has been introduced explicitly by a rigorous and formal way, so verifiable (and not less falsifiable) by its corollaries whether checking their logical consistency in mathematics or testing them experimentally in physics.

So, if the totality is granted as what contains its externality in its internality and therefore generates a fundamental doubling by itself and meant by the concept and quantity of information, time corresponds to the projection of information only on the "screen of internality" as well-ordering (including the rigorous mathematical meaning of "wellordering").

If one defines the units of that fundamental, physical and mathematical time, starting from the units of information, a bit, that unit of time can be defined in turn as the "result of a bit of information" as the mapping of a bit of information into a certain bijection (among two possible ones, totally).

That unit of time can be visualized by the record either " 0 " or " 1 " in a tape sell of Turing machine and thus by the executions of any elementary operation of Turing machine: so, it can be defined as the class of execution of Turing machine, quantum computer, and consequently by the result of quantum measurement or by the process of de-coherence in general. 
Thus, the unit of time would be to be defined fundamentally and physically as information (i.e. quantum information for the consideration is physical) being a "unit of de-coherence" (which is a volume in the imaginary domain of Minkowski space and thus, a "subluminal volume", i.e. a volume as a function of time in Euclidean space). That kind of definition implies for the unit of time to be correlative to the Bekenstein bound meaning the same after the substitution of energy by time ${ }^{3}$ (the latter is used here). Thus, the Bekenstein bound suggests that the "temporal screen" of the totality more relevant to the present research directed to mathematical history developing in time is equivalent to the "energetic screen" of the totality postulated in physics (even in quantum mechanics) by energy conservation.

The same fundamental unit of time originating directly from the totality can be defined mathematically as the mapping of Hilbert arithmetic into Peano arithmetic (Penchev 2020 August 25) determined in virtue of the "same number", i.e. by the mapping of a bit of information into a the unit ("1") of Peano arithmetic.

The totality implies the unit of time even formally and logically if one utilizes the fundamental tautology (FT) referring to the logical equivalence of the totality and a bit of information (Penchev 2020 October 20), namely:

$$
\text { "A is true" } \Leftrightarrow\{[(\mathrm{A} \Rightarrow \mathrm{B}) \Leftrightarrow(\mathrm{A} \Rightarrow \neg \mathrm{B})] \Leftrightarrow[(\mathrm{A} \Leftrightarrow \mathrm{A}) \Leftrightarrow(\mathrm{B} \Leftrightarrow \neg \mathrm{B})]\}
$$

Then:

$$
\text { "A unit of time" } \stackrel{\text { def }}{=} F T " \Rightarrow[(\mathrm{A} \Rightarrow \mathrm{B}) \Leftrightarrow(\mathrm{A} \Rightarrow \neg \mathrm{B})]
$$

Accordingly, the logical expression for the projection of the totality onto the "temporal screen" can be represented by the omission of the equivalent counterpart, [(A $\Leftrightarrow A) \Leftrightarrow$ ( $\mathrm{B} \Leftrightarrow \neg \mathrm{B})]$, on the one hand, and the reduction of logical equivalence to implication (i.e. the omission of the reverse implication linking what can be interpreted as the "totality" to what can be interpreted as a "bit of information").

Not less, the logical description of that temporal projection of the totality can serve as a logical description of complementarity in quantum mechanics: indeed, the projection cancels the dual counterpart, $[(A \Leftrightarrow A) \Leftrightarrow(B \Leftrightarrow \neg B)]$, however the cancelled expression is equivalent to the rest available counterpart, $[(A \Rightarrow B) \Leftrightarrow(A \Rightarrow \neg B)]$, which represents the "temporal screen". Thus, the temporal image of the totality understood formally and logically is the same as the totality and incomplete to the totality simultaneously, but in a consistent and perfectly faultless way. In other words, the complementary in quantum mechanics manages to express the same as Hegel's dialectics, but without any contradiction to formal logic (to propositional logic, more precisely).

This is a very important necessary condition to be established mathematical history and elucidating the necessity of a model originating from quantum mechanics: history needs the totality to be theoretical, but in a consistent way, to be mathematical. Fortunately, quantum mechanics has resolved the problem for the totality to be meant in a mathematical and logically faultless formalism and which can be borrowed and only adapted by history trying to become mathematical.

The Cartesian dualism of the modern Western philosophy and science, fundamental for its episteme ("episteme" in the sense of Foucault) and cognition even until now does not admit particularly any unification of the speculative ontology and empirical science

\footnotetext{
${ }^{3}$ That is by means of the Planck constant.
} 
furthermore experimental and based on mathematical models. They are separated by still the same abyss between the "mind" (of speculative ontology) and the "body" (of empirical science).

However, the establishment of mathematical history needs the unity of Hegel's speculative ontology of dialectics and relevant mathematical models. Quantum mechanics being an experimental and mathematized science has found a way out of Descartes's dilemma and thus, out of the modern episteme including that underlying the contemporary empirical science.

So, the present paper following the paradigm of quantum mechanics as literally as a general direction violates also the dogma for speculative ontology (particularly, Hegel's dialectic or Kant's transcendentalism in its base) not to be unified or even only considered together with any part of empirical science.

Further, still a few examples of that "heretical", non-Cartesian unification will be suggested, namely: in relation to Husserl's phenomenology (as "historical and mathematical phenomenology") as well as in relation to Heidegger's and Gadamer's hermeneutics ${ }^{4}$ (as "historical and mathematical hermeneutics").

The introduction of the "fundamental unit of time" as above, in a formal and logical way is simultaneously a relevant occasion for the "hermeneutical circle" being fundamental in hermeneutics to be considered also formally and logically in accordance with, or as a formalized speculative counterpart of the fundamental unit of time.

The hermeneutic circle understood as the "vicious circle" of classical logic is not more than a redundant tautology incapable to prove anything remaining equally problematic with or without it. If it is referred to the "excluded middle" of classical logic, Hegel's triad can be considered as its counterpart violating the law of non-contradiction (e.g. after the "excluded third" and "non-contradiction" are equivalent Boolean expressions).

Not less reasonably or formally, it can be considered as the exemplification of the other fundamental law of classical logic: the law of identity, that is as a "vicious circle", after which the premise implies the conclusion as well as the conclusion implies the premises, and thus, the conclusion and the premise are logically equivalent, i.e. an exemplification of the law of identity in the final analysis (the hermeneutical circle though notable equivalently by both premise and conclusion is identical to itself).

Here is how the fundamental tautology via the logical expression of the "unit of time" allows for the hermeneutical circle not to be interpreted as a trivial tautology and thus as absolutely redundant:

1. The hermeneutic circle exemplifies the equivalence of the totality and a bit of information just according to the "fundamental tautology" where the "totality" is meant as a hermeneutic circle, and a "bit of information" means the hermeneutical premise and conclusion.

\footnotetext{
${ }^{4}$ The expression "Heidegger's and Gadamer's hermeneutics" means as Heidegger's hermeneutics as Gadamer's hermeneutics as two separate philosophical doctrines though related to each other closely. The "father" of modern dualism, Descartes (as well as Leibnitz) being both great philosopher and great mathematician gave an example for following: how speculative ontology and mathematical theories can be developed in parallel and thus unified in a single mind.
} 
2. The fundamental unit of time can be defined equivalently so:

$$
\text { "A unit of time" } \stackrel{\text { def }}{=} F T " \Rightarrow[(\mathrm{A} \Leftrightarrow \mathrm{A}) \Leftrightarrow(\mathrm{B} \Leftrightarrow \neg \mathrm{B})]
$$

This equivalent definition is in virtue of the "FT" itself, and the conclusion, $[(A \Leftrightarrow A) \Leftrightarrow$ (B $\Leftrightarrow \neg B)$ ], can be granted as a logical definition of "hermeneutical circle" ("HC") meaning that it can be considered as the dual counterpart of the "unit of time" as the latter is defined logically by $[(A \Rightarrow B) \Leftrightarrow(A \Rightarrow \neg B)]$, i.e. by a well-ordering "step of time". In other words, the "hermeneutical circle" as a "unit of time" would correspond to the option of any of both choices as a dual unit of time; or briefly:

$$
\text { "A (dual) unit of time" } \stackrel{\text { def }}{=} F T " \Rightarrow \text { "HC" }
$$

The concept of hermeneutical circle can be visualized in terms of Turing machine so: the transition to the next tape cell is an equivalent (or dual) unit ("step") of time to the record of either " 0 " or " 1 " in the current tape cell. In other words, the execution of any single elementary operation of Turing machine defines the same unit of time in it.

\section{BOTH TRANSCENDENTALISM AND DIALECTICS ORIGINATING FROM THE POSTULATE OF THE TOTALITY}

A few previous papers (Penchev 2020 October 20; 2020 August 25; 2020 October 5; 2020 October 19) have elucidated already the way for both transcendentalism and dialectics to originate from the totality withal in the present context, i.e. as scientific (i.e. falsifiable) hypotheses rather than as philosophical doctrines "metaphysical" in the sense of Popper.

What remains is the properly historical or temporal aspect meant rather by dialectics than by transcendentalism (though it implies the former) explicitly: the historical totality to be defined in a way rigorous and similar to that of the mathematical totality (as the consistent completeness of mathematics) or the physical totality (the universe).

The historical totality moves itself by itself as dialectics makes clear. The frontier of historical time (as that of time at all) moves itself by itself forward (and never backward, i.e. time arrow is meant). However furthermore, the historical totality would be to contain its externality, which is the future, in its internality, which is the past. In other words, the historical totality as transcendental implies for the past to be doubled (unlike the frontier of the present remaining an "immovable point" in the meaning to coincide with its counterpart in definition).

However, the usual approach to history considers the past to be single and unchangeable rather than doubled in virtue of necessity to contain the future thoroughly into itself. In fact historical time is meant only as a memory, available in the present moment, of all "previous present moments" . This convention being as if an obvious postulate should be declared as "false" to the historical totality and thus, to the "mathematical history" as it is intended in the present paper.

\footnotetext{
${ }^{5}$ The expression "all previous present (1) moments as a memory in a certain present (2) moment" (as well as the motion of the immovable point of the frontier of time) is not a "Hegelian oxymoron" (or a logical contradiction) since it means a well-ordered set of different (in general) states of the same, i.e. in a formally and logically consistent way: "present (1)" and present (2) (to which "previous" is determined) are different disjunctively (as well as "motion" and "immovable" can be distinguished as defined in relevant different relations).
} 
Immediately and formally, that "second history" underlain by the "second historical time" would refer to the reversed time arrow. However, that definition is not fruitful because it is either inaccessible (by itself) or cancelling historicity (together with the "normal" time arrow). Fortunately, it can be approached indirectly by the "hermeneutical fundamental unit of time" (as above) meaning formally and logically that reversed time arrow.

In fact, hermeneutics means always a certain interpretation determined by a certain individual or group interpreter in a certain moment of the normal time arrow. The sense of that interpretation seen just according to the normal time arrow is a "value field" making certain alternative (abstractly being equally probable) of historical choices much more probable than others (abstractly being also equally probable). That field deforms any certain historical choice hermeneutically since the historical actors choose in accordance to their value system.

The mathematical description of that value field as a "second history" however following in the direction of the "first history" (that of the historical events) is a necessary condition for the establishment of mathematical history (able to predict though probabilistically, but in a rigorous way, as quantum mechanics does).

Summarizing, the historical totality is doubled just as the mathematical totality or the physical totality, or the properly philosophical totality. The image of the historical future is given hermeneutically in it: as a value field predetermining the historical choices partly and probabilistically.

\section{$\mathrm{V}$ THE ORIGIN OF THE $20^{\mathrm{TH}}$ CENTURY'S PHENOMENOLOGY AND} HERMENEUTICS FROM THE TOTALITY

One can find (rather speculatively, in an only philosophical way independent of the available empirical and historical confirmation) two elements in European philosophy even since Ancient Greek one: the totality in the dawn of philosophy in Pythagorean school and the pre-Socratics and the human being after Socrates. Both can be seen already synthesized in Plato into the "ideas" if one considers (for example) the sacral Pythagorean Number referring to the totality by itself (and thus divine and sacral for the human beings) as the Plato idea (already desacralized or "profane" once it is available in the human beings): in other words, the one element, the totality, turns out to be synthesized with the other element, the human being by means of ideas as what the totality represents within the human being.

The philosophical foundations of mathematical history needs for that synthesis to be made explicit for mathematics has to be unify with the humanitarian history of human beings possessing free will. The unification is researched by the category of possibility (and probability) shared by both mathematics and free will rather than on the base of the opposition of necessity and free will (sometimes alleged to be Kantian).

Descartes's dualism being in the beginning of the modern European philosophy added the postulate of the gap between the body and the mind, the human being and reality: an abyss in the totality making it impossible at least to human cognition, and surmountable only by God being the Totality by Himself. Thus, the Socratic "human being" is linked to the preSocratic "totality" even in a mystic Pythagorean way, but permanently, in any act of thought overcoming the abyss between the body and the mind somehow (for example by God's Help). 
Mathematical history would not be possible after Cartesian dualism for it should be situated into the inaccessible abyss of the desacralized human cognition (or God's Will of theology) therefore being not less inaccessible (or accessible only mystically).

The "Copernican revolution" of Kant's transcendentalism restored the totality already in human cognition inferring its definitive properties and postulating it implicitly by them. Hegel's dialectics and dialectics of history, particularly, appeared immediately once transcendentalism had overcome the Cartesian abyss.

Indeed, mathematical history is rather relevant to physical and mathematical dialectics than to dualism.

Husserl's phenomenology generated a few fundamental innovations in the philosophical doctrine of the totality following the general direction of European philosophy as ancient as modern. However, he did not relate his doctrine to history and historical cognition directly and explicitly: an approach and application of phenomenology to history was elaborated as philosophical hermeneutics by Heidegger and Gadamer (rather as a methodology of history by the latter).

One can demonstrate (as the present research will conjecture) that the implicit approach of Husserl's phenomenology to history and that of Heidegger's and Gadamer's hermeneutics (or an inverse "dialectics of origin" after the former) can be seen as both related and opposite, and thus complimentary in a wider sense.

For this purpose, a new kind of Husserlian reduction, historical reduction can be defined as well as well its close link to existentialism 6 once "historical phenomenon" be reduced to the present. To be justified the introduction of "historical reduction", a reinterpretation of Husserl's eidetic, phenomenological, and transcendental reduction in the context of as the development of European philosophy being a doctrine of the totality as the idea of mathematical history is necessary.

"Eidetic reduction" can be seen as a mathematical reduction as an interpretation of the fundamental and basic "transcendental reduction" therefore connecting transcendentalism to mathematics (in a way different form that of physical and mathematical transcendentalism, but presumably linkable to it) by back reflection. In contemporary terms, eidetic reduction is able to generate the "eidos" (or idea) as the class of equivalence of all interpretations of the same class, a "thing" being objectified withal starting from interpretations (even from a single one): thus, eidetic reduction postulates that interpretations implies its idea whether formally and logically or loosely (i.e. "philosophically").

Eidetic reduction can be found as the "category" of the mathematical theory of categories, i.e. as a certain mathematical structure defined axiomatically. The theory of categories investigates first of all the morphisms of categories therefore reaching to the eventual consistent completeness of physical and mathematical transcendentalism in virtue of the postulate that the theory of categories can be investigated as a category and thus, absolutely in its own framework.

However, the concept of eidetic reduction means a rather different relation to transcendentalism as the eidos can be understood as a transcendental totality containing the externality of its interpretations within itself as a necessary nucleus of any possible interpretation. Then, the postulate of eidetic reduction adds that one can obtain that nucleus

\footnotetext{
${ }^{6}$ Heidegger rejected to be an "existentialist”, for example in Heidegger @.
} 
constructively removing all variable (accidental) properties one by one in virtue of their variability, but conserving all unchangeable (essential) properties as definitive (or mathematically, axiomatic) in relation to the "eidos" (i.e. that definitive or axiomatic essence, the nucleus of properties) of the "thing" (i.e. all possible variations or interpretations adding accidental, variable properties to the "essence").

One can apply further the postulate of eidetic reduction to any real historical process in order to acquire its definitive and unchangeable eidos. An additional postulate can identify any historical event with just one property of the historical process. Then, the eidos would represent a series of definitive events available in that process wherever it occur: in any alternative or counterfactual historical process to that at issue.

Furthermore, the same nuclear series of events would be a mathematical structure in virtue of what eidetic reduction is. Summarizing, the postulate of eidetic reduction can justify the option of mathematical history studying the eidoi of historical processes as mathematical structures. However, that consideration does not suggest any method how an eidos can be constructed starting from a given history.

"Phenomenological reduction" to "phenomenon" (in the sense of Husserl's phenomenology) can be accomplished by "epoché" or the "bracketing of reality": one abandons the question whether the entity at issue is real (i.e. belonging to reality) or not. Phenomenological reduction can be understood and consequently formalized in a few different ways:

1. It removes the doubling of anything as being both in the human being (or the subject, or the "mind") and reality (or being an object, a "body") therefore reducing it to its phenomenon. The fundamental, formal and logical tautology equating the totality to a bit of information is relevant to it. Furthermore, it follows the tradition of the modern Western philosophy and particularly the mind-body problem of dualism interpreting the totality and transcendentalism in its terms.

2. However, it can be interpreted quite otherwise not less relevantly: one abandons the question whether the entity at issue is itself (among a class of entities consisting of as other existing, i.e. alternative entities as non-existing, i.e. counterfactual entities). In other words, one achieves the eidos (as that class) of the entity (belonging to the class), i.e. therefore by "eidetic reduction" coinciding with the "phenomenological reduction" in the present meaning (and unlike the former meaning " 1 ").

As to terms of mathematical history, one would be to mean a class of histories as alternative as counterfactual, to which the real history belongs. The class is definable e.g. by two historical events shared by all histories whether alternative or counterfactual, or the real one: all possible historical pathways connecting smoothly in time two events (i.e. as ordered in time) constitutes the class which would be the phenomenon (in this meaning) of the meant real history.

A fundamental difference between two meanings of "phenomenon" (respectively, of "phenomenological reduction") is obvious even still formally and mathematically: the meaning " 1 " doubles and thus generates a bijection, representable as a whole by a bit of classical information, unlike "2" juxtaposing to the one single, "real" element a set of arbitrarily many (thus, infinite in general) alternative or counterfactual entities, representable as a whole as a qubit of quantum information. 
If one need identify the two meanings, a bit of information has to be equated to a qubit of quantum information: a statement that can be proved even rigorously, formally and mathematically by the mediation of Hilbert arithmetic, and then, by its identification with the separable complex Hilbert space of quantum mechanics ${ }^{7}$ (Penchev 2020 August 25). Then, "wave function" both transfinite natural number in Hilbert arithmetic and state of quantum system would the phenomenon of the entity at issue, and therefore unifying the two meanings as follows:

"Wave function" can be interpreted as the characteristic function of a relevant probability density distribution (including Dirac's $\delta$-function particularly), the maximal value of which (corresponding to the most probable entity) would refer to the "real" entity (particularly to the single real entity if the Dirac function is the case) and this corresponds to a qubit as the value of quantum information.

Furthermore, the pair of the wave function and its dual wave function corresponds to the doubling, and thus, to a single bit of classical information, on the one hand, and to the meaning "1" of "phenomenon" acquired by "phenomenological reduction", on the other hand.

Seen so, phenomenological reduction generalizes eidetic reduction (isomorphic to the meaning " 2 ") by the doubling (corresponding to the meaning "1").

Husserl emphasized that the third reduction, "transcendental reduction" is the same as phenomenological reduction, but now one can interpret the sense of the "same" much more precisely, i.e. by the identification of phenomenological reduction and eidetic reduction as above: the meaning " 1 " related to reality (or not less to the "mind") because the totality is the "same" as the meaning " 2 " referring to a state of the totality or to a transfinite natural number in Hilbert arithmetic therefore thinkable as a (well-ordered) state of infinity.

Then, the "global space" of the totality meant by Hilbert arithmetic is the "same" as the local space of the totality meant in any entity and meant by quantum mechanics.

Consequently, the identification inferred from "transcendental reduction" implies in turn the foundations of mathematics (as Hilbert arithmetic) and the foundations of physics (as quantum mechanics) to be merged: this links physics and mathematics to be interpretable thoroughly by each other.

If one manages to prove that the foundations of history can be added to those merged foundations of both physics and mathematics, and all the three in the primary totality of the philosophy, the intended establishment of mathematical history would be achieved.

Then, "historical reduction" will mean (in definition) the "same" (analogical to that "same" linking phenomenological and transcendental reduction) as transcendental reduction but directed to history and its foundations to be seen philosophically, by the totality, but not less formally and mathematically, thus merged in physical and mathematical transcendentalism.

Particularly, historical reduction is directed to achieve a third interpretation of wave function together with the available already two ones as: (1) transfinite natural number; and

\footnotetext{
${ }^{7}$ Physical and mathematical transcendentalism implies that a properly physical equivalent of that equation of a bit to a qubit is necessary to exist, and this is the Schrödinger equation, the basic one for quantum mechanics: one conjecture partly justified in previous papers (Penchev 2020 July 10; 2020 August 25), but still awaiting in the queue of future research to be articulated explicitly in detail and rigorously, formally and mathematically.
} 
(2) a quantum state according to quantum mechanics. That interpretation should mean the exhaustive description of all the class of counterfactual or alternative histories to the real history: mathematical history is possible only to that class immediately, and to the real history only by means of it.

Speaking loosely, one can say that the way for history to be mathematized is opposite to that of natural science: the real history can be delivered from its mathematical model by "taking away" alternative and counterfactual histories unlike the reality of any natural science which is "more" than its mathematical model and can be achieved starting from it only adding new and new accidental properties to the model, an infinite set of accidental properties in general.

That approach for history to be mathematized, made this so difficult and opposed to that of natural science, can be realized also introducing a hypothetical viewpoint (and a corresponding observer attachable to it) external to the totality, i.e. as if "outside of it". Indeed, the totality in definition contains an equivalent counterpart of it, however within itself.

Anyway, the external observer as the observer's counterpart within the totality and the actually internal observer can be distinguished by what each of them would see as the relation of model and reality. The reality for the inherently internal observer would be "more" than any mathematical models needing an infinite set of additional properties in general to be achieved, but "less" to the internal twin of the "transcendent observer", i.e. ostensibly "outside of the totality".

That reality less than its model would be a certain history, i.e. a series of anything, but well-ordered in time necessarily as successive temporal states of that "anything". Thus, the model, if that is the case, would be the class of all possible histories.

In comparison with that necessary innovation for history to be mathematized, what the inherently internal observer utilized any mathematical model to the observed reality would see is that, on the contrary, reality is all possible models.

Those two alternative statements can be unified as "ontological invariance" meaning that the change between "external observer" to the totality and "internal observer" within it is equivalent to the change of the relation of "reality" and "model" to the relation of "part" and "whole": that is "reality" is "part" to "model" as to "external observer", but "model" is "part" to "reality" as to internal viewpoint.

The latter worldview has been standard until now, but it does not allow for history to be mathematized in any way. This is possible only after the change of Gestalt to the former worldview and meant in the present paper in the framework of the generalization consisting of both.

One can notice that the worldview of quantum mechanics is situated exactly on the border of both worldviews. This is proved as the well-known theorems of the absence of hidden variables in quantum mechanics (Neumann 1932; Kochen, Specker 1968)@ interpretable also as the coincidence of "model" and "reality" being definitive for quantum mechanics. Obviously, the coincidence of "model" and "reality" is just the common border shared by the class of all classical ontologies being "convex" to any model and nonstandard being "convex" to any reality (i.e. "concave" to the model). This makes clear, particularly, why the mediation of quantum mechanics (being on the border) is necessary for delivering mathematical history. 
The historical reality (meaning mathematical history as its background) corresponds to the fundamentally random single measurement of quantum mechanics and empirically accessible only by the readings of the so-called apparatus. If one had involved a nonstandard quantum mechanics describing the "apparatus" (unlike the standard one studying the microscopic quantum entity rather than the apparatus), it would be isomorphic (i.e. equivalent mathematically) to mathematical history.

An analog of the many-worlds interpretation of quantum mechanics as to mathematical history would be possible and might be called the many-models interpretation of mathematical history: each model of them would generate an alternative history. The last observation can serve as a narrative link to the discussion of Heidegger's and Gadamer's hermeneutics in terms of the sketch above about historical reduction withal mathematical: any model can be interpreted as a state of the "value field" predetermining historical choice partly and probabilistically in general.

To define "philosophy as a rigorous science" (in fact, in relation to the paradigm of logic and mathematics as rigorous sciences), Husserl introduced the temporal "stream of consciousness", to which philosophy once being rigorous should refer. That rigorous philosophy is to order necessarily well (i.e. as a well-ordered temporal series) the unordered atemporal and aspatial logical and mathematical truths (or theorems as phenomena) in virtue of what consciousness is: a stream.

The "eternal" logic and mathematics (or the "eternal" philosophy, on the other hand) are reflected on the stream (temporal) screen of consciousness as the intended "rigorous philosophy". By the way, that "rigorous philosophy" would be to be falsifiable, particularly, once it claims to be verifiable (as rigorous). Then, the mentioned physical and mathematical transcendentalism, physical and mathematical dialectics, historical and mathematical phenomenology or hermeneutics, etc., all of them being falsifiable definably, would be to be considered in the area of rigorous philosophy, i.e. by means of the same stream of consciousness implying their fundamental falsifiability.

History meant standardly is a temporal series always. Historical reduction (as introduced above) is an interpretation of the abstract and general transcendental reduction to history, and thus, implies the unity of "historical eidos" and "historical phenomenon" as to any temporal series meant by history as usual.

That "historical phenomenon" originating from the transcendental doubling after a kind of "historical epoché bracketing reality" (which means historical factuality or counterfactuality) is temporal inherently.

Unlike it, "historical eidos" introduces the set of all real and counterfactual histories as a single element utilizing relevantly mathematical concepts for mathematical history. Then, the mentioned historical unity of both eidos and phenomenon can follow from the fundamental mathematical identity of any set (for "historical eidos") with all elements of it (for historical phenomenon).

However, any history being certain as a temporal series adds additional choice(s) and information due to the choice of a certain element among all the elements of the relevant set. 
That choice is predetermined by the "temporal screen" and its "profile", , on which a certain historical eidos is "projected".

Following Husserl's track, one can think of the "temporal screen of history" as the "stream of consciousness of the transcendental subject" being the projection of the "eidos of transcendental history" after the specific "screen profile" (for example that of Europe or China, etc.). That generalized screen is "curved" by a common, social or civilization value field "deforming" all the historic choices according to its preferences without complete predetermination, though.

That social or civilization value field can be inferred in a few independent ways distinguishing the present extrapolation of Husserl's phenomenology to history from Heidegger's or Gadamer's philosophical hermeneutics, after which the former can be interpreted as a kind of "transcendental hermeneutics": a single, common hermeneutics of history interpreting itself by itself or realizing itself in an admissible Hegelian reading of Husserl's phenomenology applied to history.

That transcendental hermeneutics originates from "transcendental and historic invariance" meaning an application of transcendental invariance to history, namely the equivalence of any historical process to an atemporal eidos referable and thus shared by all the class of alternative and counterfactual historic pathways, to which the given real historic process belongs. The choice of the historical process at issue among the class needs additional information, for delivering which the value field is defined.

Abstractly, it can be considered as reverse causality originating from the future and directed to the past determining additionally the standard causality, in terms of which would be to be represented as fundamental randomness or "God's intervention" in human history: a relatively insignificant factor along all the human decisions, however, causing the bifurcation of historical process in branches and following one of them.

Hermeneutics discusses alternatively the same factor determined additionally that historical process to necessity by fundamental randomness, however interpretable as reverse causality in the framework of "historical phenomenology" (as here):

The unrecognizable "Will of God" visible as fundamental crucial coincidences of historical processes is substituted by human decision justified by a certain state of value field in the investigated historical moment. The acting reverse causality of "God's Will" is replaced equivalently by human decisions in virtue of transcendental and historical invariance. That course of thought interprets the fundamental randomness of historical process as a human decision as if irrelevant to the essence of historical process, i.e. insignificant and without realizing its aftermath to historical bifurcation. Anyway, that insignificant decisions correspond to value field in that historical age as any human action of any person not being "crazy".

One can see continuity smoothly transforming human decisions in "God's Will" and vice versa as well as any causality as predetermination, but only post factum, for only the happened fact allows for a certain causality to be understood as predetermination unlike many other causalities not influencing historical process. That substitution can be realized only post

\footnotetext{
${ }^{8}$ That "profile of the temporal screen" can be visualized by curving the "screen" therefore deforming the "images projected on it".
} 
factum because it means always change the reverse causality into causality and thus implies the reversion of time arrow.

Until now, the leading viewpoint was that of historical phenomenology, but it can be reversed in turn and not less smoothly to that of historical hermeneutics:

The key concepts relevant to the present context are "interpretation" and "hermeneutical circle". Any historical entity (such as a historic fact, event, process, etc.) makes sense only as interpreted though the interpretations can be quite different in general. Thus, "interpretation" can mean as the class of all interpretations as any certain interpretation. The former can be called "abstract interpretation" or "transcendental interpretation", and the latter, "concrete interpretation" or "phenomenological interpretation".

"Historical phenomenology" achieved by a Husserlian approach as above is directed rather to the transcendental aspect of interpretation (though equivalent to its phenomenological aspect in the final analysis after the identification of transcendental and phenomenological reduction), i.e. to the historical eidos atemporal, universal or "eternal", seen or visible only from "God's viewpoint": the meaning of history as in Frege's "Bedeutung".

From the "human viewpoint" inherently empirical or experimental, the same historical eidos is observable always and only as exemplified temporally, i.e. as an actual course of history clearly distinguishable from any other as well as possible, alternative or counterfactual pathway by the participation of the observer in the former case and the nonparticipation and indifference of the observer being external definitively in the latter case: in other words, the historical eidos once the viewpoint is changed to human is historical phenomenon.

Heidegger's and Gadamer's hermeneutics mean rather historical phenomenon though equivalent to its eidetic aspect in the final analysis after the identification of transcendental and phenomenological reduction. Historical phenomenon and historical eidos are linked and identifiable to each other also by the interpretation of the totality as itself by itself definitively, in virtue of being namely the "totality", in which the abstract and transcendental interpretation, on the one hand, and the concrete and phenomenological interpretation, on the other hand, are merged.

Hermeneutic circle restores historical eidos in any historical phenomenon by adding the reverse time of interpretation (and correspondingly, the reverse causality of interpretation), after which any historical entity is interpretable in the present moment being definitively in its future.

From the viewpoint of propositional logic (being atemporal), hermeneutical circle is a "vicious circle" adding at best a redundant tautology to the chain of syllogisms, and thus, "cut by Occam's razor". The viewpoint of propositional logic delivers (or it is able to deliver) only eidos and therefore, historical eidos in particular.

On the contrary, hermeneutic circle inherently temporal delivers historical phenomenon as well as also phenomenon at all (in Husserl's meaning) after the being is temporalized (as in Heidegger's "Sein und Zeit").

The concept of a bit of information may reconcile the viewpoint of propositional logic and that of hermeneutics (respectively, the redundant tautology of vicious circle and the synthetic and informative postulate of hermeneutic circle) in virtue of the fundamental, 
formal and logical tautology, particularly able to temporalize the totality by cyclicity (i.e. by the cyclic transition to a single bit of information).

The concept of hermeneutical circle means that tautology, and thus, representable as a single bit of information, e.g. as follows:

Hermeneutic circle can be considered as a specific hermeneutic (or philosophical) "unit" introduced only in order to notate the totality formally as a common measure of the totality itself and any part of it. "Measure" here (i.e. relation to "hermeneutic circle") is used rather loosely, or philosophically, meaning that the totality definitively (in virtue of being namely the "totality") represents a single quality shared by itself and anything within it. Thus, the philosophical concept of measure means only that the "totality" by itself implies the existence of an universal and general quality "measurable" just as a certain quality by the conventional unit of "hermeneutical circle".

However, any shared single quality transferred to be empirical or experimental implies to be a quantity as well. On the other hand, the same transferability as to "hermeneutical circle" seems to be problematic (at least at first glance) as far as the totality should be neither empirical nor experimental, and God's Viewpoint is granted to be fundamentally different and incommensurable with humankind's. However, the concept of common measure, just the "hermeneutical circle" implies for them to be, on the contrary, commensurable.

Anyway the same philosophical commensurability is not necessary to be interpreted furthermore as implying the existence of a certain, empirical or experimental quantity. The latter would be to be adopted as an additional axiom in the framework of, or equivalent to historical and mathematical transcendentalism summoned to establish mathematical history formally and logically, i.e. consistently.

Once the existence of that quantity shared by the totality (whole) and part be admitted, it can be identified as information in virtue of the obvious statement that the two quantities sharing the same unit are in turn the same as well: one need prove that the philosophical unit of hermeneutical circle (postulated to be transferable to a relevant quantity) is isomorphic to bit of information (by the way, the same statement is what is meant by the "fundamental, formal and logical tautology"). Anyway, the isomorphism at issue can be demonstrated independently, e.g. as follows:

Hermeneutic circle unifies indistinguishably fact and interpretation as a historical entity as well as the normal and reverse time arrows: conventionally, the time of fact can be accepted to be "normal", and, that of interpretation, "reverse" therefore implying the option for them to be dividable disjunctively. Then, the unity of fact and interpretation, on the one hand, and the distinction of fact from interpretation, on the other hand, constitutes together a bit of information? .

Heidegger's hermeneutics can be distinguished from that of Gadamer rather conditionally by the emphasized aspect: fundamental and referring to the totality rather after Heidegger, and properly historical and referring to the interpretable historical fact eventually after Gadamer. The latter author explicated the unity of both approaches.

The specific interest and fruitfulness of Gadamer's hermeneutics can be generalized by the investigation of "value field" predetermining in a certain degree both historical decision

${ }^{9}$ One can demonstrate the same isomorphism by a tape cell of Turing machine. For example or conventionally, the cell notates 'hermeneutical circle', the record of " 0 ", 'interpretation', and "1", 'fact'. 
and interpretation though possibly in different ways as to any concrete historical fact. Thus, the reverse "time of interpretation" is inaccessible empirically in the being of historical fact by the "time of historical decision" collinear the "time of fact" granted to be the "normal time arrow", and thus, absolutely cognizable not only post factum.

That fundamental innovation of Gadamer and its influence as to the establishment of mathematical history will be discussed in detail in Section VIII.

VI TO TRANSCEDENTALISM, DIALECTICS, PHENOMENOLOGY, AND HERMENEUTICS DEFINED RIGOROUSLY AND SCIENTIFICALLY

All the mentioned philosophical doctrines originated from the Western philosophical category of the totality in turn arisen to resolve the Cartesian dualism. Anyway, the latter was conserved in the dichotomy of philosophy (on the "mind" side) and science (on the "body" side). Husserl's idea to transform "philosophy as a rigorous science" does not mean to transfer the philosophy from the "mind" side to the "body" side of experience and experiments, but on the contrary, to unify them by the concept of the totality, i.e. applying once again the concept of the totality to the relation of philosophy and science rather than only to the philosophy itself. In other words, though the intention for philosophy to become a rigorous science seems to be anti-metaphysical to, and naturalizing philosophy at first glance, its essence is just opposite: to make science philosophical and metaphysical.

As Husserl as Heidegger tried to elucidate the fundamental philosophical meaning of "time" by its relation to "consciousness" or "being". The former, being a mathematician by education and initial profession, kept closer links of philosophy to logic, mathematics, and science. The later rather opposed them calling Husserl to blame him in "naturalization", i.e. restoring their dualism therefore cancelling the intention of phenomenology to unify them by means of the totality. In fact, Heidegger's time not less than Husserl's time was related to the totality (as Heidegger's being), but it was considered in a way inconsistent and even incommensurable to time in science therefore involving (in "Sein und Zeit") a new existential conception of abstract and philosophical human experience accessible inherently and initially, self-obviously to human beings and then, absolutely different to empirical experience and experiments in science. That new kind of experience, introduced philosophically, was necessary to reconcile it with to the totality in order to be able to be fundamental namely in the "fundamental ontology".

However, that solution shifted the Cartesian abyss within the experience itself dividing it into philosophical experience in fundamental ontology and empirical experience in science. That approach is not relevant to the objectivity of the present investigation.

On the contrary, the solution is researched rather in Husserl's direction and even on its radicalization to the interpretation of philosophical doctrines (such as transcendentalism, dialectics, phenomenology, and hermeneutics) as scientific hypotheses or theories therefore falsifiable whether formally and logically or experimentally. "Philosophy as a rigorous science" seems to mean just that, at least philosophically, metaphorically or as a wish.

Childishly or naïvely, the intention now is just this and literal: the quoted philosophical doctrines to be considered as scientific theories explicitly in a rigorous, formal and logical way on the base of relevant definition of the totality:

The totality is meant as a meta-philosophical category interpreted and exemplified by different names and complemented specifically in transcendentalism, dialectics, 
phenomenology and hermeneutics. Furthermore, it is defined formally as that extremity, thus opposed to all being not extremal, which contains its externality in its internality, or doubling anything by itself. The boundary between the externality and internality is "immovable' to doubling therefore needing or admitting special consideration.

The totality defined formally so (but not "formally" in a rigorous and logical meaning) is prepared rather to be included in axiomatic tuples (or semi-axiomatic, such a set of principles in physics) to be redefined, or more preciously, to be defined additionally by the specific relations established in all other items in the corresponding tuples ${ }^{10}$.

Only after that and only in that sense, corollaries both falsifiable and verifiable can be inferred and checked whether for logical contradiction or experimental refusal.

In an absolute rigorous meaning, falsifiable statements are only the items in the formal tuples. The corresponding ideas meant implicitly or contextually in the axiomatic systems are "falsifiable" only in a loose sense being intermediate between the falsifiable scientific theories and the metaphysical philosophical doctrines.

The philosophical doctrines can be considered as an "eternal archive" of ideas and opposed to the scientific theories falsifiable in any certain historical moment. Those two "poles" suggest an intermediate area between them, each element of which would be to be defined as a class (or union) of falsifiable theories so that the refusal of one or more (but not all) of them does not mean the refusal of the class itself.

\section{HISTORICAL DIALECTICS REFORMULATED BY INFORMATION AS AN APPROACH TO MATHEMATICAL HISTORY}

Hegel applied his doctrine of dialectics (by the way, Marxism borrowed it) to history to explain it by itself: by the self-motion of the World Spirit or Reason in History (the change of Marxism is rather cosmetic: the substitution of the World Spirit by the "matter"). The world and history change by themselves being forms of the totality. So, Hegelianism (particularly Marxism) implied and justified social and political radicalism.

The discourse of theoretical history was introduced. A general narrative of Progress in history appeared. However, it was not scientific, but quasi-scientific at best. It should replace the usual formal logic inherent to science into "dialectical logic" able to state for any proposition to be true (at least, from the viewpoint of formal logic). It was not predictive, but descriptive and only post factum, able to explain any event or process, once it has taken place, "dialectically" (in virtue of dialectic logic able to prove any proposition as necessary from the viewpoint of the usual logic and scientific method). So the value (if any) of historical dialectics was rather ideological than scientific.

Historical dialectics in its original form is inapplicable to mathematical history and even inconsistent to it. Fortunately, that crucial disadvantage can be neutralized by the concept of information for Hegel's "triad" (as "hermeneutic circle" above) can be interpreted formally and logically as a bit of information under the specific condition for the definition of bit to be reversed: the "direction of bit" is that of any choice, and the "direction of triad" is reverse. Then, a bit represented by a tape cell of Turing machine can visualize the triad so: " 1 " notates Hegel's "thesis"; "0", "antithesis"; and the empty tape cell, Hegel's "synthesis".

\footnotetext{
${ }^{10}$ The "totality" is meant as an initial and fundamental notion definable only contextually in the consistent and complete tuple of a certain axiomatic system. In advance, it can be rather suggested as an approximate idea, to which the tuple at issue would refer particularly.
} 
One can think of the relation of "bit" and "triad" furthermore so. If one applies a bit of information to itself, its dual counterpart would be just the triad. The "fundamental, formal and logical tautology" describes and notates a bit of information consistently to formal logic. One admits the self-reference of a bit (i.e. to itself) just in virtue of the same tautology for the meta-level of the totality coincides with the level itself as far as the meta-level as external to the totality has to be contained in it definitively, and thus, necessarily. Furthermore, the tautology identifies a bit of information with the totality and consequently, a meta-bit of information, with a bit of information.

Once the self-reference of a bit of information has been justified, this implies immediately the consistent formal and logical definition of "triad" as the dual counterpart of a bit of information in the framework of the "meta-bit" (admissible and justified as identical with a bit of information). Then the discourse of mathematical history consistent formally and logically will be the dual counterpart of Hegel's dialectics of history: a discourse descriptive, post factum, and inconsistent to formal logic and scientific method.

Hegel's self-movement of history by itself, indeed, is inconsistent to formal logic as well as any change, including mechanical motion, by itself, i.e. considered as a whole or a form of the totality. It can be "repaired" to be consistent to formal logic only by a wider context, in which it is a part of other and more general whole.

Fortunately, it can be introduced even to the totality itself (to which no more general totality to be "more total") by means of the triad as the dual counterpart of a bit information and being self-referential consistently to formal logic in the virtue of the fundamental tautology.

Speaking loosely and rather metaphorically, one might say that the self-movement of history from the human viewpoint can be transformed consistently to formal logic as the dual counterpart of God's viewpoint to it, furthermore inherently consistent to both formal logic and mathematics and allowing for the establishment of mathematical history.

\section{ANOTHER APPROACH TO MATHEMATICAL HISTORY: PHILOSOPHICAL PHENOMENOLOGY AND HERMENEUTICS IN THE FOUNDATIONS OF MATHEMATICAL HISTORY}

Philosophical hermeneutics and phenomenology contributes essential new approaches to mathematical history after reformulating dialectics by information consistently to formal logic and scientific method. Using the formal scheme of Hegel's triad above, "hermeneutical circle" can be seen formally once again as both "meta-bit" and "bit" identical to each other in virtue of the definitive property of the totality, particularly containing its meta-level in itself, i.e. identifying "meta-level" and "level". Then, one can think of the Hegelian triad and hermeneutic interpretation as equivalent due to sharing the dual counterpart of a bit of information: indeed, the "time of interpretation" (introduced above) is reverse to the "time of fact" (ibidem), and the former coincides with the "time of triad", i.e. from "thesis" via "antithesis" to "synthesis". Indeed, both are post factum meaning a fact of the past whether interpreting it or synthesizing it.

The same formal equivalence of triad and interpretation allows for the triad to be realized as the class of all possible interpretations of the historical fact at issue meant as Hegel's "thesis" in the "dialectic logic of development" inconsistent to formal logic allowing for 
contradiction, therefore violating the law of "non-contradiction"11. If one chooses a certain interpretation or is supplied by an unambiguous rule to choose an element among all the class of triad (interpretations), the "ideological" (and quasi-scientific at best) viewpoint of dialectics, post factum, can be changed into the scientific one following the "time of fact" exante rather than the reverse time of interpretation.

That crucial step for transforming Heidegger's hermeneutics into a scientific method of theoretical history was realized rather by Gadamer than by Heidegger himself. It transforms the speculative "hermeneutic circle" (unusable by scientific method directly, analogically to Hegelian triad, for redundancy, though not for inconsistency, to formal logic) adding the empirically accessible "field of value" following the scientific time of fact and determining the historical actors' interpretations however not univocally.

Mathematical history adopts the contribution of Gadamer's hermeneutics absolutely only complementing it by the natural postulate that the value field can be described mathematically withal exhaustively. That additional postulate needs and links "historical phenomenon" originating from Husserl's phenomenology, but furthermore representable by a relevant mathematical structure for the objectivity of mathematical history. It involves the identity of "meta-bit" and "bit" in a still one way: rather as the identity of "meta-qubit" and "qubit", or equivalently as that of "global space" and "local space" after identifying "phenomenological reduction" and "eidetic reduction" in virtue of the concept of "transcendental reduction".

Then, the identity of "meta-qubit" and "qubit", or respectively, of "local space" and "global space" would be to be understood so. The choice of a certain phenomenon among all phenomena and the choice of an eidos among all eidoi is the same, mathematically isomorphic, and containing the same quantity of information being necessarily generalized, namely as quantum information and thus measured in qubits.

The concept of quantum information being relied on that of infinity (more precisely, on the concept of infinite set or infinite series) allows for not distinguishing between the local level of space and the global level of space, or respectively, between "level" and "metalevel". It is invariant to the transition between them after the identification of them to antiisometry in virtue of transcendental invariance.

As a result, the hierarchy of level and meta-level is removed and substituted by their duality and idempotence: in other words, the level is the meta-level to its dual level as the meta-level to the level, as well as vice versa. Duality and idempotency remove also the wellordering of level hierarchy therefore and particularly admitting a finite model of infinite (respectively, of infinite series or infinite sets).

One can visualize and exemplify the above observation by type hierarchy in Russell's theory of types as follows. The type hierarchy unlimited and ad infinitum in principle implies for the set of all types to be infinite. The same type hierarchy is reducible to two dual levels

\footnotetext{
${ }^{11}$ The present approach to formalizing "dialectic logic" does not follow those of any paraconsistent or non-classical logics revising or generalizing implicitly or explicitly the "law of non-contradiction". Both formal logic and "non-contradiction" particularly are conserved literally, but dialectic logic is referred to a specific logical structure called a "bit of information" and defined by the fundamental tautology. Then, the triad of dialectic logic is defined formally as the dual counterpart of a "bit of information" and in virtue of the rigorous and logical definition of the totality.
} 
conventionally notable as "level" and "meta-level" mutual to each other, and thus, idempotent.

The concept of transcendental reduction able to appear as "historical reduction" and identifying phenomenological reduction and eidetic reduction means the same kind of identification of level hierarchy ad infinitum to only two, but dual levels as the exemplification of, therefore visualizing, type theory.

In turn, the applicability of historical reduction to mathematical history can be illustrated by the generalized invariance in the foundations of mathematics, after which an arithmetical variable able to accepts different values ad infinitum is identifiable as a logical variable accepting only two but dual values therefore idempotent (to each other). Indeed, the class of equivalence of all level hierarchies can be identified with the set of all natural numbers and thus implies (without being equivalent to) all the natural numbers in Peano arithmetic ${ }^{12}$. Then, Boolean algebra isomorphic to propositional logic is isomorphic in turn to Peano arithmetic (Penchev 2020 August 25) after the condition for the two-valued logical variable to be identifiable with the arbitrarily many-valued arithmetical variable meant in the mathematical model of historical reduction in particular.

The "global" history, i.e. seen retrospectively, post factum, actually ended and as it is usual in the discourse of the science of history, would be to be identified in the "local" value field in any historical moment determining absolutely or partly (i.e. probabilistically) historical choices possible or done then and there. Historical reduction being an aspect of transcendental reduction after involving time, temporality, and historicity explicitly means just that identification of the temporal global history with the atemporal (as well as aspatial) local state of value field in any certain "point" of history.

The same observation inspired by the establishment of mathematical history can be articulated otherwise as well. There exists necessarily just one counterfactual history (which can be eventually and particularly available as a real, but alternative history) which is equivalent to that atemporal and aspatial value field however well-ordered by the quantity of probability of any given alternative in the fan of all possible ones as to a certain historical choice.

A perfectly new worldview of history is involved: the real history can be considered as the universal and transcendental history valid anywhere and omnipresently, whenever and forever once any counterfactual history (to that real one) is available necessarily as given locally by the state of value field of a certain historical moment. The real history to be realized or understood globally runs through all possible counterfactual (to it) histories however given locally (also aspatially and atemporally in the sense of the zero-dimensionality of a "point" what a historical moment is once it is thought mathematically). Thus, any counterfactual history, accessible neither empirically nor experimentally (as written inherently in "conditional mood" ostensibly "forbidden" for history), is transformed anyway in a phenomenon accessible at least empirically and even experimentally studying a certain state of value field.

\footnotetext{
${ }^{12}$ All natural numbers are finite in virtue of the axiom of induction (featuring Peano arithmetic from set theory): indeed, " 1 " is finite; " $n$ " is finite' implies that " $n+1$ " is finite'; then, the axiom of induction is applicable to the property of a natural number to be finite and implies for all natural numbers to be finite. On the contrary set theory does not include the axiom of induction, but it contains the "axiom of infinity", which is not available in Peano arithmetic.
} 
The sketched deduction represents furthermore the formal and logical link between Husserl's phenomenology via the explicated here idea of theoretical and historical phenomenology to Heideger's hermeneutics and at last, to Gadamer's:

Husserl's phenomenology implies historical reduction as transcendental reduction, starting from the premise of the deduction, and Gadamer's hermeneutics postulates any historical fact as interpreted inherently and definitively by a state of value field whether post factum, i.e. posteriori (the value field of the historicist(s) studying the fact at issue) or exante, i.e. a priori (the value field of the actor(s) creating history). The concept of value field originating from Gadamer's hermeneutics is involved right to identified the form and the latter mathematically, i.e. as isomorphic to each other.

The formal and logical link between the premises of historical phenomenology to the conclusion of historical hermeneutics consists in the identification of all counterfactual histories and all states of value field inspired by "historical reduction" (as it is introduced here). The same identification implies involving quantum information as embodying the relevant mathematical isomorphism as to mathematical history.

IX ALTERNATIVE OR COUNTERFACTUAL HISTORY TO REAL HISTORY: THE IDEA OF TRANSCENDENTAL AND UNIVERSAL HISTORY

Any history written in the discourse of "conditional mood forbidden for history" is a counterfactual history. At least one essential historical choice has resulted in an alternative different from its counterpart in the real history and then, in an alternative chain of causal changes creating a branch of history parallel to the real course.

One of the most famous examples is Philipp Dick's “The Man of the High Castle" usually qualified as a masterpiece of the sci-fi novels therefore asking the fundamental question of counterfactual discourse: literature (fiction) or science (reality), and answering it convincingly in favor of the former; thoroughly in the framework of literary fiction and thus, out of science representing reality rather than fiction.

An essential and maybe crucial counterargument offers Robert Fogel's "Railroads and American Economic Growth: Essays in Econometric History" (1964) ${ }^{13}$ won the Nobel Prize in economics (1994) for "for having renewed research in economic history by applying economic theory and quantitative methods in order to explain economic and institutional change" 14 .

The implicit philosophy of history in his research (not only in this work, but also in others, e.g. Time on the Cross, 1974) is "collinear" and "commensurable" with the approach to mathematical history, which generalizes his contribution to cliometrics in a sense:

Both cliometrics and mathematical history would be to be relied on the universal and transcendental history as eidetic as phenomenal both in Husserl's meaning. Only that understanding of philosophy as a single one as many parallel ones is able to unify the standard scientific discourse of history "post factum" and the a-priori mathematical discourse "exante" therefore generalizing temporality to transcendentality.

\footnotetext{
${ }^{13}$ One might admit that "The man in the High Castle" (1964), or at least its cultural and historical background in the USA, might influence "Railroads and American Economic Growth: Essays in Econometric History" (1964).

${ }^{14} \mathrm{https}$ ://www.nobelprize.org/prizes/economic-sciences/1993/press-release/ (accessed on 22.11.2020).
} 
History turns out to be necessary, and the mathematical comparison of the real branch to a certain counterfactual branch demonstrates this statement by means of the approximate conservation of a quantity constructed for the research, namely "social savings". Then it, attributable to railroads, can be calculated and shown to be insignificant $(1.3 \%)$ to the counterfactual case without them, and substitute technologies might compensate or even exceed the influence of railroads measurable by the "social savings" at issue.

The deep philosophical idea of Fogel's research can be seen, interpreted or generalized from the worldview of mathematical history so. Mathematics introduces always quantities able to unify different qualities. One can interpret two or many branches of history (particularly, one of them tcan be the real one) as two or more corresponding qualities and following the mathematical approach, to be unified by a relevant mathematical quantity ("structure" in general and more precisely). This is just the idea exemplified by "Railroads and American Economic Growth" so much successfully to win the Nobel Prize.

Mathematical history can be inspired by Fogel's triumph explicating and justifying the idea itself, only implicit in the "Railroads...".

One can notice even the "family resemblance [Familienähnlichkeit]"15 of Fogel's approach and the "fundamental, formal and logical tautology" definitively linking a "bit of information" and the "totality":

For example and following Hegel, one can speak of the "dialectics of quality and quantity", and after that, see it formally and logically as a bit of information. Indeed, any two or more qualities are absolute different and separated from each other, but not less and simultaneously, they are the same quantity therefore distinguishable as different amounts of it, but sharing its single quality, namely to be that quantity at least, if there is no other word for their unity.

Then, a bit of information (along with all other possible interpretations) is able to notate formally and logically the elementary transformation of quantity into quality (more exactly, the unity of quantity of two equally probable properties, i.e. qualities, in each of them disjunctively) just reversely to Hegel's triad directed to depict the opposite transformation of quality (i.e. at least two different qualities) onto quantity. After that, the "totality" already implicitly involved by a "bit of information" in virtue of the "tautology" turns out to be embedded in that dialectics of quality and quantity:

A particular corollary or exemplification of the dialectics of quality and quantity (formalized as above) represents Fogel's approach to unify two histories (namely, the real

\footnotetext{
${ }^{15}$ One should distinguish rigorously, formally and logically "set" from "family resemblance": the former grants the unity as a new entity, namely "set", the latter does not do this. Thus, their set is a shared and definitive property of all elements, regardless of any relations between them; on the contrary, "family resemblance" is only relations between the elements, and those relations share in turn only one or more family resemblances. "Family resemblance" needs an interpreter who sees it (therefore admitting other who does not). "Set" does not need any interpreter, or speaking conventionally, one might say that needs an absolute single interpreter such as "God". The transition from "family resemblance" to "set" means, on the one hand, the mathematization itself of history, in which the multiple interpretation inherent and definitive for history, its discourse and cognition is overcome by the class of relevant interpretations, constructing a relevant unity and set, to which mathematical history makes sense; on the other hand, a single, abstract and universal, as if "transcendental" observer and interpreter of history appears to be necessary as to history for the establishment of mathematical history.
} 
one with railroads and the counterfactual one without railroads) by a relevant mathematical quantity (or structure, in general), namely "social savings". Mathematical history (or "cliometrics" by Fogel and others' term) properly should be related to the quantity of social savings interpretable as an amount in the real history and as another (in general) amount in the specially built counterfactual history, however turning out to be almost the same as the former as a result of the research.

In fact, the cognitive and research scheme of Fogel has been utilized many years ago, but rather unexpectedly ... in Einstein's special relativity. The family resemblance of them can be shown up by the mediation of Bachtin's "chronotope":

The alternative history can be defined as a counterfactual history under the definitive condition to be real one, but somewhere else. For example, the history of China is alternative to that of Europe. Then and following the standard paradigm of science of history, two discourses of histories (of Europe or of China) appear naturally, each of them supplied by its own "chronotope" as any discourse. Well, special relativity generalizes that even the physical discourses of two regions (reference frames) and thus their "chronotopes" would be different under the postulate of speed light in a vacuum. However, it establishes a new quantity, namely "space-time interval" or "space-time distance" to be invariant after the transition from a reference frame to another, or respectively, between "physical chronotopes".

Fogel, analogically to the space-time interval of special relativity, introduced the quantity of social savings to unify the chronotopes of both real and counterfactual history.

Fogel's problem and successful solution (so successful to be noticed by the Nobel Prize of economics in 1993) is particular, anyway, though tracing the pathway to the most general problem and solution discussed here and related to the establishment of mathematical history withal universal.

If Fogel decided how two certain and quite restricted histories, one of which real, and the other counterfactual, to be unified by a specially constructed quantity, the maximally general problem would refer to that mathematical structure ("structure", in turn, as a generalization of "quantity" in the contemporary mathematics) able to unify any class of historical pathways, some of which counterfactual, others real (though alternative to each other), and thus the class of all possible histories, but determined unambiguously, e.g. by two events shared by all historical trajectories belonging to that class.

The separable complex Hilbert space is the structure researched and advocated in this work. It can be seen equivalently, furthermore, as a certain quantity, quantum information corresponding to Fogel's "social savings" in a sense sketched above and generalizing it as much as possible, i.e. to the totality.

The history able to be identified as mathematical history is generalized to the ultimate extreme, to the totality. Following the metaphor already coined, history can be observed as mathematical only as if from "God's viewpoint" (or physically, from "God's reference frame") as if out of the totality. However, that trope restricted by transcendentalism and dialectics weather philosophical or historical and scientific might mean only the totality itself as the invariant of transcendentalism being both immanent and transcendent on the boundary of them:

Mathematical history is the history of the totality, and thus, merging to the history of the universe, i.e. to cosmology and physics, therefore able to share with quantum mechanics the 
same mathematical structure, the separable complex Hilbert space, and the same quantity, quantum information.

Along with the dialectics of quantity and quality involved a little above, a cyclic dialectics of both extremes appears: for example the extreme of the totality including all definitively and the opposite extreme of a bit of information, but coinciding with the former in virtue of the fundamental tautology withal formally and logically after it. That "cyclic dialectics" synthesizing the opposition of both extremes recollects Nicholas of Cusa's doctrine or that of Giordano Bruno.

As to mathematical history properly, it suggests as if what science observes by and within quantum mechanics as if externally to the totality would be to be the same as observed by and within mathematical history as if internally. They coincide only on the boundary of the totality, where both extremes can be identified definitively. However, what is outlined so is the foundations of mathematical history thus referring the absolute generality of the totality. Specific research in mathematical history restricts it to the particular generality of a relevant historical whole therefore enriching the initial mathematical structure with additional essential properties (axioms) or, on the contrary, simplifying it by means of classes of equivalence. Anyway, the former is the rule, the latter are rather exceptions.

\section{$X$ THE IDEA OF FORMAL AND MATHEMATICAL HISTORY BY THE PARADIGM OF QUANTUM MECHANICS: THE "FEYNMAN HISTORIES"}

Once quantum mechanics and mathematical history have shared the same separable complex Hilbert space as foundations, any language (i.e. “interpretation”, e.g. Feynman's) of the former can be both adapted and adopted by the latter.

Feynman's one is rather suitable and fruitful for it can be used not only by quantum mechanics and mathematical history (meant as human and social history only), but by the mathematical theory of any evolution in addition involving the mathematical model of evolution tree.

The present section, however, will be restricted only to the applicability of Feynman's viewpoint to mathematical history.

The base is the identification of historical pathway with Feynman's trajectory, due to mathematical isomorphism. If one considers an infinite set of those trajectories between two certain points of any kind of vector space including the usual three-dimensional space of experience, in which human history takes place, with a corresponding probability density distribution defined on the continuum of all of them, the characteristic function of that distribution is a wave function, i.e. an element of the separable complex Hilbert space. It describes all the infinite class of possible trajectories as a single complex trajectory by two independent variables: a universal time during each of them and relative (density) probability to happen, being a parameter of each of them and thus, a variable definable on the all set of them.

One might say (but only quite conventionally and following Feynman's visualization) that time is a variable, and probability is a "metavariable". However, the converse statement exchanging which is the variable and which is the metavariable, i.e. the level and meta-level in an idempotent way, is not less correct: after exchanging in parallel both dual spaces, one would fetch the initial wave function.

The original Feynman visualization is impossible already in the latter case, but another not less interesting visualization is relevant: that of the "many worlds" language and 
interpretation suggested by Hugh Everett III. The possible Feynman trajectories are exchanged by the real, but alternative Everett worlds.

However, what about "probability" transformed from a "metavariable" to a "variable"?

Well, probability, though it is often interpreted as the quantity of possibility, is not more than any normed quantity seen only and properly mathematically, i.e. such as velocity in special relativity, where it can be normed always by the fundamental constant of light speed in a vacuum and less than a unit just as the standard probability whether "objective" or "subjective". So, the metavariable of relative probability turns out to be relative velocity involved by special relativity, and what is a trajectory in the Feynman interpretation is again a trajectory in the Everett interpretation though the variable and metavariable are exchanged mutually.

Anyway, but only at first glance, the difference is conserved as to the sense of the metavariables in each case: the Feynman trajectories are only possible unlike the Everett worlds, which are only real.

One can equate an Everett world to a reference frame in general relativity (since the relative probability, resp. relative velocity is variable) therefore implying a force field, in which the "probabilistic field" turns out to be transformed. The parallel worlds ostensibly non-interacting can be consistently unified as a class of reference frames sharing the same space and thus interacting in virtue of the force field at issue.

Looking deeper, the alleged difference between Feynman's and Everett's approach vanishes absolutely for the quantity of probability density in the former can be analogically interpreted as the quantity of subluminal velocity (to the light speed constant). So, both approaches admit equally well an equivalent representation where both variable and metavariable can be real and actual separately, but mutually complimentary as well as the case of the one actual, and the other be possible (probabilistic).

If both variable and metavariable are real and actual, the Feynman and Everett interpretation are idempotent to each other in a Hamiltonian meaning: the variable and metavariable are the two Hamiltonian conjugates (e.g. momenta and space coordinates) whoever exchanged to the opposition of level and meta-level in each of them (e.g. momenta are variables in Everett's, but metavariables in Feynman's, and vice versa as to coordinates).

If the one is real and actual (never mind which), and the other is probabilistic (again never mind which), this involves Max Born's interpretation of quantum mechanics, and thus, of the separable complex Hilbert space $^{16}$ (after which wave function is interpreted as the characteristic function of the probability density distribution of the variable or that of the idempotent metavariable in the dual space).

Thus, the Feynman interpretation (as far as it is directly interpretable in terms of mathematical history after the substitution of "trajectory" with "historical pathway" or

\footnotetext{
${ }^{16}$ The qubit Hilbert space involved by the theory of quantum information unifies all the three interpretation:

a property inherited from the separable complex Hilbert space in virtue of the isomorphism to it under an additional, but natural condition. Thus, the quantity of quantum information measured by the units of qubit represents the phenomenological "epoché" to reality, but now, in terms of quantum mechanics and information, which is an obligatory condition for the foundations of mathematical history in order to be able to borrow the mathematical formalism of the separable complex (or qubit) Hilbert space.
} 
"history") is able to promote also the applicability of the Everett and Born approaches as well as the concept of quantum information for realizing the establishment of mathematical history.

First of all, one need make clear how to relate variable and metavariable in mathematical history and to think of them to be idempotent:

The natural variable is time or historical time: it is shared by the real, counterfactual or alternative history. Unlike the special relativity, it can be granted as universal, and thus the same in each of them. However, the corresponding metavariable seems not to be any quantity, but a specific quality different in each historical pathway. For example that quality can be designated by the name of the region for an alternative history to take place (e.g. the history of China) or by the initial and bifurcating one or more facts dividing a counterfactual history from the real one. One would acquire a set of names (furthermore interpretable as properties and thus qualities, each of which attributable unambiguously to each pathway): quite insufficient for any quantity of metavariable to be introduced. One needs an additional and intermediate mapping from the set of natural names of histories into a relevant set consisting only of mathematical entities such as numbers, vectors, probabilities, etc.

However, the naturalness of that auxiliary mapping seems to be quite and even fundamentally problematic as far as it originates from a definitively non-empirical entity what the totality is. In other words, the naturalness should be interpreted in a way not being empirical in definition, but referring to history, an empirical science, only by the mediation of the totality postulated for the establishment of mathematical history to be possible.

Indeed, the totality is able to choose unambiguously a relevant metavariable to the variable of historical time, respectively a mapping of the set of names of histories into mathematical entities. Even more, it can justify an empirical counterpart of it, thus accessible experimentally.

The mediator is the conception of transcendentalism (here interpreted rigorously and in a falsifiable way: historical and mathematical transcendentalism).

Mathematical transcendentalism (Penchev 2020 August 25) delivers the equivalence of the well-ordering "theorem" and the axiom of choice (almost trivially provable in set theory) as a conclusion of the postulate of the totality as well: therefore allowing for mathematization of humanitarian sciences such as history consistent to the totality (as Hegel's philosophy of history demonstrated first by his "dialectics").

Then, historical time corresponding to the well-ordering "theorem" implies a hypothetical counterpart after the equivalent axiom of choice, which is interpretable as information and can be specified as quantum information (i.e. the information of infinite set) as far as that counterpart as a quantity is measurable by the units of elementary choices such as bits or qubits.

The counterpart of historical time can be explicated so: it represents the quantity of elementary choices necessary for the set consisting of "names of histories" to be ordered well to be equivalent to the corresponding historical time being well-ordered in definition. Then, once that quantity is measured by the units of elementary choices, it is necessary to be information or isomorphic to information.

Information in general (particularly quantum information) is the metavariable conjugate to the variable of historical time after the above abstract cogitation. However, what would be 
to be its historical sense is unclear yet. The usual scientific discourse of history does not involve it explicitly, so that it should be a specific contribution of mathematical history:

If one considers any historical process not as current, but post factum just as historical cognition describes it as happened in the past, an exactly determined quantity of information necessary to be that process ordered in time, in the way it has taken place really, corresponds to the variable of historical time, obviously being a natural metavariable needing to comprise and comprehend all the process as a whole. Then, this is the historical sense of information as the searched metavariable: the information containing in the narrative able to represent the process at issue relevantly.

One sees immediately why the usual scientific discourse of history is fundamentally incapable to introduce it: it is metavariable just to it, thus needing it to be complete, so being not introducible in it currently, in its course, implying for it to be self-predicative. On the contrary, mathematical history is able to define it rigorously just in virtue of involving the totality in advance as a postulate therefore predetermining the consistent resolution of the problem of self-predicativity to the totality definitively.

Still one feature is to be noticed in relation to information as an explicit metavariable of mathematical history: it reflects that any historical fact is interpreted inherently to exist; in other words, historical facts which are not interpreted do not exist, they make sense only in a certain historical discourse or narrative. Of course, different historians would create different discourses and narratives as if sharing the same facts though interpreted differently in general. From the viewpoint of mathematical history, they would be equivalent to alternative or counterfactual histories ${ }^{17}$.

Following the intention of Gadamer's hermeneutics, one can suggest for any historical discourse or narrative, being definitively post factum, to correspond to real historical actors' counterpart, by which they are able to choose one or other action or decision. However, that counterpart does not share the converse time of interpretation, but the "normal" arrow of historical time.

Formally in mathematical history, the exchange of variable and metavariable is embedded in the counterpart, more precisely described so: the variable is the previous metavariable, the discourse or narrative information available for historical actors as value "field" partly predetermining their actions and decisions. However then, historical time turns out to be the metavariable able to "continue" further or to the next moment of historical time only after a certain decision or action has been accomplished (just corresponding to being a metavariable properly).

The interpretation of mathematical history in terms of quantum information would mean just the understanding of the qubit Hilbert space as the information weather of the discourse, narrative or of the relevant value field conditioning historical choices or actions and being complimentary to each other as opposite temporally: the former time arrow is "backward", post factum, that of interpretation of the historical process as complete; and unlike the latter of historical time being "forward", ex-ante.

The local space of historical time seen as global space comprises all states of value field in any moment. Quantum information allows for that value field to be represented

\footnotetext{
${ }^{17}$ One can distinguish the alternative narratives from counterfactual narratives to the real history in the meant sense so: alternative narratives are facts in the real history, they have taken place; the counterfactual narratives, on the contrary, are not facts in the real history, they have not happened.
} 
mathematically regardless of its interpretation in a certain history. The dual viewpoint by means of interpretational time, "backward", exchanges variable and metavariable, local and global space: now the discourse (narrative) is situated in the global space containing as local spaces all possible interpretations in all alternative or counterfactual discourses (narratives).

The concept of transcendental or universal history inferable from mathematical history would suggest just the above dual viewpoints to be idempotent: if not, an implicit global value field would act therefore conditioning for "action" (or "action time", i.e. historical time) and interpretation (or "interpretational time") not be equivalent to each other differing only by the direction of time arrow. Furthermore, that implicit global value field can be identified as a certain local value field in a exactly determined moment of historical time, in which meta-discourse of transcendental or universal history (as in the present paper) would be involved; and vice versa: the postulate of ideological neutrality would imply for the two dual mathematical histories to be idempotent (i.e. as if from "God's worldview").

"Born's interpretation" of mathematical history (i.e. that analogical to Born's interpretation of quantum mechanics) would equate both descriptions of history either by actual historical time and virtual discourse (narrative) or by virtual (i.e. counterfactual) historical time, but actual discourse, on the one hand, with its description as transcendental or universal history as above involving quantum information and the qubit Hilbert space. Speaking loosely, the following example would be relevant for the sense of Born's interpretation of mathematical history:

The narrative of The Man of the High Castle describes a counterfactual history certainly. However, if it could be formalized mathematically, i.e. to a certain wave function representing exhaustively all information containing in the novel, it is the same wave function as that of a real history to any eventual description of it. In other words, in the pole of mathematical history seems that Philipp Dick's fiction has described a real history: by the way, a suggestion implicitly claimed by the author by means of the novel.

The many-worlds interpretation would involve the global history as follows. All possible counterfactual histories would be granted as alternative, i.e. really happened, but in alternative universes, at least, and at best, as alternative history somewhere around the world. That latter case might postulate conventionally zero probability of all alternative histories "taken place" out of the earth (i.e. in the cosmos). So, the global human history (only within the earth) would be the transcendental and universal history for any other history has turned out to be impossible, with zero probability to occur. However, an objection would be that many alternative histories taken place on remote planets orbiting around other stars different than the sun are unknown, but real.

\section{BOTH "FEYNMAN HISTORY” AND QUANTUM MECHANICS SHARING THE COMMON UNDERLYING STRUCTURE OF THE SEPARABLE COMPLEX HILBERT SPACE}

The Feynman approach to mathematical history reveals the isomorphism with quantum mechanics therefore challenging common sense in many relations. The most of them are well-known from quantum mechanics, and now, only transferred into mathematical history via the formalism of Hilbert space.

However, there exists still one referring to the relation of mathematical history and quantum mechanics, and not explicated in the usual ways for the latter to be interpreted. 
Quantum mechanics means microscopic physical entities such as elementary particles, atoms, etc., and history (and particularly mathematical history), macroscopic bodies such as humans, weapons, ships, planes, military technics, etc. obeying classical mechanics (the properties, relations and laws of which are quite different from those of quantum mechanics).

The trouble can be represented relevantly by the quantity of energy, the conservation of which is a fundamental physical law valid in both, on the one hand, and quite different in magnitude in the microscopic world of quantum mechanics in comparison with the macroscopic history, on the other hand. The quantity of energy and its conservation implies for the "big" and "small" to be quite different withal in an absolute sense.

On the contrary, the shared mathematical formalism suggests for them to be equivalent even physically. This is a contradiction, fortunately, resolvable easily, but to detriment of common sense: the absolute difference of the "big" and "small" is to be suspended, and proclaimed to be relative (even generalizing Einstein's relativity to a new kind of "external" or "discrete" reference frames) therefore restricting energy conservation to make sense and thus be applicable and valid only to "temporal physics" and heralding the non-temporal physics of quantum information: the phenomena of which are out of space and time by themselves, and only "projectable" on the "screen" of space-time. The conserved quantity in that generalized case is quantum information, not energy. A new, generalizing law of quantum information conservation comprises both old, temporal and new, non-temporal physics.

Mathematical history is to be related to the latter: a statement seeming to be paradoxical for history is temporal definitively, and mathematical history in particular would be to be temporal as well. In fact, this is only reason for the mathematical history not to be established until now since this which is possible, on the contrary and speaking figuratively, only from the "eternal (i.e. non-temporal) viewpoint of God".

Anyway, the non-temporal viewpoint to history is necessary only as a "Wittgenstein ladder" to be established mathematical history, i.e. in its foundations. One need define and then identify the local and global space of history interpreted always and definitively. Fact ant interpretation are divided disjunctively, but in a dual and idempotent way between the two spaces. The "eternal viewpoint to history" introduces the pair of fact and interpretation as necessary for mathematical history; however being dual to each other, only one of them is available actually therefore keeping the temporality of history in each case whichever it be. So, the eternality of history is available explicitly only in the foundations, but implicit in mathematical history as established and articulated.

The worldview after mathematical history is really challenging to common sense: speaking loosely or figuratively, what one sees in quantum experiments is ... our history and we ourselves or at least entities isomorphic to our history and we ourselves. The huge part of information is lost in mathematical history to be able to be identifiable with quantum mechanics after sharing the qubit Hilbert space.

However, that information lose is conditioned still by the course of time, or more precisely, by the principle of maximal or increasing entropy. What can be conserved is only quantum information or the classical information of infinite series or sets, and thus, equitable to the former. All classical information referring to finite series or sets is being lost in the course of time therefore determining decreasing information or respectively increasing entropy. 
However, information seems to increase (respectively for entropy to decrease 0 in human history due to the ordering activity of humankind. This is a possible objection to mathematical history (at least, to the way to be established here) and thus it needs a relevant explanation. In other words, the contradiction appears after linking physics (particularly quantum mechanics) where entropy increases to history being human activity where information increases, and entropy decreases. What is the source of inflow information in history, furthermore defined in a way not to contradict to physics? ${ }^{18}$

The short answer is: it is due to the historical choices according to value field and corresponding to any interpretation, necessarily accompanying any fact in a narrow sense in order to be transformed into a historical fact properly, i.e. interpreted, recorded, and registered in a relevant discourse (narrative). This means that mathematical history is to distinguish the vanishing classical information from the conserved quantum information by the criterion for information to be interpreted, i.e. only ,written down on paper”, by which an implicit interpretation is chosen already.

Quantum information in physics can be considered furthermore as that classical information (thus taking place in a certain space-time spot or "point") which is "written down on paper in the Book of Nature" (thus omnipresent instantly, but interpretable anywhere by means of four additional "letters" of classical information propagating only subluminally) ${ }^{19}$. All unrecorded classical information vanishes in time, all recorded classical information is quantum information being conserved. Thus, information can only decrease in time (and respectively, entropy increases therefore justifying the "second law of thermodynamics"). The viewpoint of mathematical history sharing the same formalism complements a new understanding of "written down history" to physics, in fact, available already in it by itself ${ }^{20}$.

The same solution of the problem as to mathematical history can be divided in successive stages and discussed in more detail:

1. Quantum information is only the recorded classical information. This means that the record (and thus implicit interpretation) adds a complimentary doubling of a certain part of what takes place, i.e. to the fact to be transformed into a historical fact. The other part of information, which is not registered, vanishes in the "sands of time" increasing entropy by itself $^{21}$.

18 The analogical inflow of quantum information justifies dark matter and dark energy as this is demonstrated in: Penchev 2020 October 20.

19 This statement is proved in the theory of quantum information and known as the law of teleportation.

20 The so-called Hawking paradox claiming that any black hole cancels quantum information (therefore violating that law of conserving it) is a very famous example that the idea and problem about the recording of information has appeared in physics before that of mathematical history as here.

21 The corresponding understanding of the "record in the Book of Nature" is very instructive. Measurement in quantum mechanics is the record in question. It cancels necessarily just the half of information of the measured quantum coherent state therefore transforming quantum information into finite classical information (what any result of measurement represents) by neglecting the second, dual part of finite information. That measurement as a record can be equivalently transformed into an explicit decision of the experimenter which half of variables be measured (recorded) and which not. Though the measurement occurs here and now, i.e. in a certain space-time point, it can be considered as omnipresent after complementing with "four letters" (respectively, with two bits or a single "complex bit": "complex bit" is different from "quantum bit, qubit") able to restore the information 
The doubling is dual in virtue of the time arrow of interpretation (record), post factum, and thus conversed to that of factual time. Each time arrow (either that of fact or that of interpretation) is disjunctive to other. The two "times" cannot be given simultaneously temporally, i.e. "at the same time" literally. If both are available (as in quantum information), this implies for well-ordering to be cancelled as well as any projection on the temporal "screen".

That understanding of quantum information as the recorded classical information is mediated by two logical equivalences: (1) quantum information is equivalent to the classical information of infinite series or sets (Penchev 2020 July 10); (2) the classical information of infinite series or sets is equivalent to the classical information of two disjunctive ("gapped") finite series or sets (Penchev 2020 August 25). If both premises are valid, here is complemented only the interpretation of the two "gapped" pieces of classical information as referring either to fact either to interpretation (record).

2. The interpretation (record) of fact is transformed into (particularly, identified as) the corresponding historical decision predetermining (at least, partly) the fact at issue, and the time arrow of which is collinear with that of the fact. However, the decision being ideal and the fact being material can be considered as "gapped" not less than the opposition of fact and interpretation. In turn, the decision unlike the record being material just as the fact does not conserved: it represents that classical information vanishing in the course of time and conditioning increasing entropy.

So, that transformation (or identification) of record into decision generates a complex invariance consisting of two elementary oppositions neutralizing each other applied jointly: (1) the conversing time arrows; (2) material (conserving) versus ideal (vanishing). The same invariance can be understood or realized so as well: the time of interpretation converted into the time of decision implies for the latter to be only virtual (ideal) rather than real as that of the fact.

\section{HILBERT ARITHMETIC TO MATHEMATICAL HISTORY: NEOPYTHA- GOREANISM}

The "Feynman history" explores the isomorphism of quantum mechanics and mathematical history. A previous paper (Penchev 2020 August 25) discusses the unity of the foundations of both quantum mechanics and mathematics also sharing the separable complex (qubit) Hilbert space. Then and as a conclusion, mathematical history possesses the same foundations as mathematics: thus, a form of neo-Pythagoreanism heralding that ontology, or "first philosophy" is mathematical, even arithmetical though in a new, generalized meaning, as Hilbert arithmetic.

Concisely, "Hilbert arithmetic" is defined (in the same paper) by a few properties or relations:

lost after measuring anywhere in the universe by doubling the available finite classical information of the measurement. However, those "four letters" as well as the classical information of the measurement itself separately can be transmitted only subluminally. Then, one can grant that quantum information is transmitted instantly everywhere, by the record of the measurement occurring here and now, but nonetheless omnipresent, being verifiable everywhere. Of course, measurement in a narrow sense is only a human activity, however it can be generalized by the concept of decoherence not needing humans to take place, and thus, "writing down in the Book of Nature" not worse than us. 
1. It consists of three Peano arithmetics: two twin, and idempotent complementary copies of Peano arithmetic ${ }^{22}$ in its standard interpretation as well-orderings; the third one is a nonstandard interpretation $^{23}$ of it possessing the structure of Boolean algebra and thus as isomorphic to propositional logic as consistent to set theory (unlike the standard interpretation after the Gödel incompleteness theorems in 1931), and even isomorphic to it after a few natural conventions.

2. Each of both interpretations seen from the other one represents transfinite ordinal numbers well ordered by the function successor as it is defined in the other twin and alternatively to the own definition. Then, those transfinite ordinal numbers can define equivalent "transfinite natural numbers" unambiguously. In other words, the twin Peano arithmetic contents all transfinite natural numbers of Peano arithmetic, but ordered well "backward", reversely to each other in both twins. Then Hilbert arithmetic can be seen in two equivalent, but complimentary ways: (1) as consisting of two dual Peano arithmetics; and (2), as consisting of two dual transfinite Peano arithmetics.

3. One can construct bijection of the separable complex Hilbert space and the dual one, on the one hand, and the two dual transfinite Peano arithmetics, on the other hand, after involving the Dedekind, set-theoretical finiteness. This means that any transfinite natural number can be identified as just one wave function (and vice versa as well), i.e. any quantum state being properly physical can be interpreted as a transfinite natural number being properly mathematical mapped into each other unambiguously. Mathematics and quantum mechanics (thus physics) are merged by the concept of Hilbert arithmetic equivalent to the separable complex (or qubit) Hilbert space.

4. Hilbert arithmetic can be interpreted immediately and equivalently in terms of information including quantum information. Indeed, any namesake (better, "number-sake") natural numbers, whether finite or transfinite, of the three Peano arithmetics constituting Hilbert arithmetic can represent a bit of information. Any transfinite natural number can represent a qubit of quantum information (under a formal convention) as well as the set of all bits of three number-sake elements.

The concept of information, respectively quantum information allows for mathematics and quantum mechanics (thus physics) to merge their foundations: a common informational base is divided in two branches: mathematics and quantum mechanics (physics). The latter adds only the postulate of the equivalence of the quantities of information and physical action by the fundamental Planck constant.

\footnotetext{
${ }^{22}$ They differ from each other in the interpretations of two Peano axioms. The function of successor is defined as usual in the one: $n+1$, but as $n-1$ in the other one. Then, the unit of the former is the standard " 1 ", but that of the latter is " $\omega$ " defined as the least countable ordinal. This implies that the complementary Peano arithmetic needs its twin for the unit " 1 " is involved in the latter; otherwise, as " $\omega$ " as " 1 " should be postulated in the former therefore necessarily involving an additional axiom not available in the original Peano axioms.

${ }^{23}$ The nonstandard interpretation (1) interprets the function successor as " $n=$ ", i.e. 2 means " $1=1$ ", 3 means " $1=1=1$ ", etc., and (2) unifies both complimentary standard interpretations therefore being extended from " 1 " to " $\omega$ ", which furthermore can be linked cyclically to each other due to " $1=\omega$ "

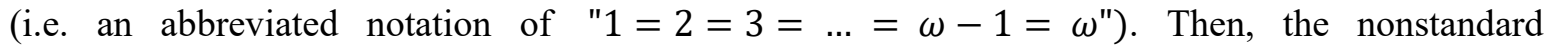
arithmetical addition turns out to be Boolean disjunction, and multiplication, Boolean conjunction.
} 
Meaning that concise representation of Hilbert arithmetic, one can notice that its interpretation by information (as in 4) can be interpreted directly in terms of the "Feynman history", and thus, in those of mathematical history for both Feynman history and Feynman interpretation of quantum mechanics share the separable complex Hilbert space as their common mathematical formalism, in turn equivalent to the qubit Hilbert space of Hilbert arithmetic.

Then, a rigorous, formal and logical link even in the form of deduction can be tracked from Hilbert arithmetic as a neo-Pythagorean kind of ontology (as fundamental as specific and historic) to mathematical history.

The main conclusion is that mathematical history suggests or even implies a contemporary doctrine of Pythagoreanism.

\section{THE TOTALITY OF BOTH HISTORY AND MATHEMATICS AND THE PRINCIPLE OF LEAST ACTION IN PHYSICS}

One can generalize historical and mathematical transcendentalism originating from the totality similarly to physical and mathematical transcendentalism withal sharing the same mathematical formalism also originating from the postulate of the totality.

Then, the shared concept of the totality and mathematical formalism are sufficient to promote a law of mathematical history similar to that of least action in physics though invalid in quantum mechanics: the law of least choice. It means that the real historical pathways is chosen in the fan of variations under the criterion of least historical choices, or that supplying most historical continuity (respectively, least social and political revolutions). It will be deduced in detail from the law of quantum information conservation in the next section.

Here and again extremely concisely, the "first deduction" in a previous paper (Penchev 2020 October 20) will be represented for it is a premise of that in the next section (which can be considered also as an interpretation of the "second deduction" in the cited paper in relation to mathematical history).

The concept of the totality by means of scientific transcendentalism whether physical and mathematical as in the previous article or historical and mathematical as it is interpreted here consistently to the context implies the conservation of quantum information after the following few logical steps:

1. The totality (by mathematical transcendentalism) implies the consistent completeness of mathematics which can be proved once the foundations of mathematics has been identified as Hilbert arithmetic. This needs the relevant kind of Pythagoreanism, which includes the world (particularly, the world meant by history as its subject) in the scope of mathematics even in a narrow sense. The link at issue was tracked in the previous section.

2. Then, Hilbert arithmetic implies the conservation of quantum information. Any arithmetic including Hilbert one is both atemporal and aspatial, and valid anywhere in the universe in definition. The same statement can be interpreted otherwise as well: the arithmetical unit is conserved universally. However, Hilbert arithmetic considers it as a bit of information or complimentarily, as a qubit of quantum information. If arithmetical unit is the same anywhere in the universe, this sameness is valid necessarily for qubit in virtue of the postulate of the totality of mathematical and physical transcendentalism.

That universal sameness of qubit means the conservation of quantum information for the representability of quantum information at all (i.e. any wave function) as a single qubit in 
virtue of the equivalence of both quantum information and single qubit to the information of infinite series or sets (Penchev 2020 July 10).

3. The branch of physics originating from the fundamental and shared foundation of information and quantum information is stood out by postulating the quantity of physical action as the dual counterpart of the physical quantum information is identified with the mathematical quantum information of Hilbert arithmetic as the omnipresent quantum information originating from the totality directly.

One should emphasize that the concept of action if it originates from the physical quantum information is not committed to the space-time framework of physics as it is common in it until now thoroughly. In other words, "action" equivalent to information suggests the existence of a new kind of atemporal and aspatial physics and thus obeying the conservation of quantum information rather than that of energy for it is out of the "temporal screen" obligatory for the contemporary physics including the Standard model ${ }^{24}$.

However, that statement (3) is to be modified for the objectivity of mathematical history by the substitution of "physical action" with "historical action" though the latter is only another interpretation of the mathematical structure (or quantity) underlying both of them.

The concept of historical action means as that of physical action the option of the atemporal and aaspatial historical actions. Historical action can be divided in two components, or two kinds of historical choice: (1) empirical, accomplished by historical actors in the course of historical event in their chronotop (their "here and now") and depending on their free will; and (2), the welded value field independent of their free will and partly predetermining the possible choices of the actors possessing free will. The latter does not accomplish by them, but rather obeys them, obliges them in advance: they ought to do one or another ethically and imperatively.

Though the physical action out of space-time seems to be rather paradoxical to physics committed to the empirical framework, the concept of historical action out of space-time is quite natural for the choice depends on mental (thus, atemporal and aspatial) conditions in default.

Then, one traces a direct, formal and logical pathway from the law of least action in physics via the conservation of quantum information to a conjectured law of least action in history for example so as it follows in the next section.

XIV THE CONSERVATION OF QUANTUM INFORMATION APPLIED TO MATHEMATICAL HISTORY: THE LAW OF LEAST CHOICE

If one applies the way in which the law of least action can be considered as generalized to the conservation of quantum information, but in the reverse direction and as to mathematical history, the hypothesis of least choice can be formulated so. The first stage (1) is to be the detailed investigation of the way how the former is related to the latter my means of energy conservation being a corollary of following the "trajectory of least action", and simultaneously, a conclusion from the conservation of quantum information therefore determining the relation between them. Thus, the first stage can be subdivided into two ones in turn:

\footnotetext{
${ }^{24}$ The "second deduction" in the cited paper (Penchev 2020 October 20) infers dark matter and dark energy from the conservation of quantum information just as the projections of the "dark" reality behind the "screen" of space-time on it.
} 
1.1. How the law of least action implies energy conservation (in classical mechanics):

Once the framework (or "screen") of space-time has been involved as necessary for the law of least choice to make sense, the first derivative of action to time, i.e. energy is constant during all the trajectory defined by the condition to be least along it. The so-called first Noether (1918) theorem of conservation once interprets that conclusion to the Lie group of time translations and the conjugate (to action) conservation of energy, and then, twice, generalized it to any pair of physical quantities conjugate to each other in relation of action.

So, an implicit premise in the law of least action is that just one single trajectory has been determined as the real one, and that single trajectory is furthermore defined by the least integral of action along it.

1.2. How quantum information conservation implies energy conservation (in quantum mechanics):

Energy conservation in quantum mechanics is a corollary from the property to be unitary being postulated in it due to the mathematical formalism of the separable complex Hilbert space:

Its vector interpretation embedded in Heisenberg's matrix mechanics and its function interpretation in Schrödinger's wave mechanics are equivalent only under the condition of unitarity therefore being a necessary for the former and latter to be unified in the contemporary quantum mechanics.

As to the projection of physical phenomena on the "temporal screen" (not being necessary in general, for example, for those of quantum information such as entanglement), it can be generalized to physics at all (as far as it is valid in its base, quantum mechanics), and further, interpreted philosophically, as the "transcendental invariance" of the temporal projection with the correlative atemporal physical phenomenon in turn complemented by a unique mathematical structure properly.

Quantum information conservation regulates the unambiguous way in which the apparatus and the measured quantum entity can correlate to each other to keep the wave function as the same (i.e. the wave function of the quantum entity by itself and the wave function reproduced by a series of measurements of the apparatus). Both extremes of that correlation can be considered as the two dual energy conservations in quantum mechanics (unlike the single one in classical mechanics due to the "transparency" of the apparatus in it). They are: (1) the invariance of energy of any quantum entity regardless of which apparatus has measured its energy (2) the invariance of energy of all the class of unitarily transformed quantum states as to a certain apparatus ${ }^{25}$, due to which the class in question can be considered as a single physical entity.

Thus, the deduction of energy conservation from that of quantum information means extremes just as the principle of least action, but the ontological status of the corresponding variations is different: relative (i.e. in relation to the correlative variation) in the former, but absolute in the latter (for the correlative interpretation is zero as far as the apparatus is "transparent" identically and definitively).

Once the kind of generalization in (1), i.e. from both (1.1) law of least action and (1.2) quantum energy conservation to energy conservation, has be made clear, then the quantum

${ }^{25}$ The unitary transformation of quantum state make sense in general only to a certain apparatus. Anyway the concept of unitary transformation can be generalized granting a certain non-unitary transformation as unitary conventionally. 
information conservation can be transferred and thus established in mathematical history in virtue of the shared and underlying structure of the qubit Hilbert space: at last another quantity or structure isomorphic to the law of least action in physics can be indicated in mathematical history and after that interpreted in history (in the next section).

That analogue of the law of least action in physics as to history and mathematical history is called the law of least choice. Speaking figuratively (and following the metaphor coined by the authors of the free will theorems in quantum mechanics in Conway, Kochen, 2006; 2009@), the "commodity of free will" in both physics and history is so valuable that it has to be saved or spared as much as possible, respectively spent minimally. That "thrift" is just the condition for the pathway of real history to be that of least choice as needing least free will. The rigorous, withal formal and mathematical meaning of that metaphor, i.e. (2) the reverse deduction from quantum information conservation in mathematical history to the law of least choice in history, can be subdivided also in two stages therefore involving the idea of still one analogue (that of energy conservation), which can be called conventionally and provisionally the "conservation of historical continuity", in turn a corollary from the conjectured law of least choice. Both are:

2.1. How quantum information conservation in mathematical history implies that conservation of historical continuity

In fact, the conservation of historical continuity means the dual analogue to that of energy conservation in physics: the smooth, steady, uniform, and collinear course of time only in one direction ("time arrow") meant in the "Lie group of translations in time" of Noether's first theorem (1918). So, the dual twin in history, unambiguously implied by the conservation of historical continuity, would be to be called the "conservation of reality" meaning that the historical pathway share the same reality along and during it, in all moments of time.

Of course, if reality is a single one in definition, the above condition is satisfied trivially. However, that is not the case in quantum mechanics shared in quantum information as well. Many parallel worlds in both have become obvious after Everett's interpretation, and the conservation of reality does make sense as a restriction as heuristic as obligate: any mix of parallel realities is not admissible.

The shared mathematical formalism transfers the same conservation of reality onto mathematical history as that along and during any possible history whether real or alternative, or counterfactual therefore implying for it to conserve the own single continuity.

The condition of many realities or many histories being meaningful doubles the variations and thus their extremes. The classical "least action" means one single variation of trajectories, but not the correlative variations of the apparatuses for it is zero in definition after all possible apparatus are "transparent" identically. Quantum mechanics elucidates that the latter variation is not less meaningful that the former.

Quantum information borrows that doubling and transforms into mathematical, indeed seeing it as the following two interpretations also dual to each other: (1) the variation of the states of value field to a single history granted to be the real one (as in the classical case); and (2), the variation of histories to a single state of value. At last, (3) one can demonstrate that any combination of both (what the concept of entanglement in the proper theory of quantum information means) can be reduced equivalently and relevantly in historical terms either to (1) or to (2). 
As far as (1) is just the classical case of the standard historiography (though eventually mathematized), quantum information conservation in mathematical history as the conservation of historical continuity can be not less equivalently and relevantly reduced to:

2.2. How the law of least choice implies the conservation of historical continuity

Any choice means a leap for the next state of history being discretely separated from the present one and can be achieved in many future real pathways, each of which possible and probable in accordance with the value field in the moment of historical choice at issue. It predetermines the choice but only partly needing the free will of the actors to be accomplished ultimately.

Backwardly and post factum realizing it, one can notice that it has conserved historical continuity whatever have been. The human free will is necessary crucially to link the "broken history", "cut time" therefore proving itself as a necessary condition for history even to exist.

Nonetheless, ex-ante, the bridge of historical continuity is neither obvious nor the continuation of history is guaranteed: the choice can turn out to be so wrong to cease the history of all the class of actors making the choice, immediately or in the close future. Not less vice versa: any historical extinction of a group of actors or its subjugation, infusion and melting into another is preceded by a series of wrong choices therefore not being able to overcome the gap of history (what any historical event is in fact), but ceasing and finishing the own history.

The human community questioned by history which the correct choice is answers according its value field summarizing the total available experience of that group. Historical memory has conserved the kind of successful decisions of similar problems sharing family resemblance to the current one as one or more relevant values possibly contradicting to each other therefore needing the free will of the community as an arbiter between them.

Even more, the actors (especially in Modernity) are not committed to follow the welded value field, but they are able to create new values or legal norms ad hoc to justify any choice contradicting the tradition. The capability of innovation is heralded a special kind of metavalue specific to the contemporary epoch therefore obeying, or politically contrary to tradition as radicalism to conservatism:

Radicalism determines a wider "window" of admissible historical and political choices, therefore deviating more from the most probable choice, and conservatism, on the contrary, shrinks it. Conservatism governs, as a rule, in the periods of continuity, in which the past predetermines the future crucially, even causally, and essentials choices or changes are not necessary, most probably even harmful.

And vice versa: radicalism dominates during "revolutions", historical discontinuity meaning the necessity of fundamental innovations due to very changed or forthcoming to change circumstances. Then, the law of least choice implies or might be interpreted as that historical pathway as less radical as possible. Radicalism seems to be a "necessary evil" admissible if and only if it is proved to be necessary: conservatism does not need any justification, but radicalism does always.

However, the reverse error is much more often in the real human history: conservatism continues to dominate regardless of the obvious necessity of changes, due to which they are delayed sometimes fatally, causing the extinction or obedience of human communities.

The law of least choice claims to supply the historical actors with an objective criterion for the necessity of change, withal quantitative, formal and mathematical. 


\section{INTERPRETATIONS OF "LEAST CHOICE"}

The concept of "least choice" means the quantity of information or quantum information, to which all possible situations of uncertainty resolvable by decision can be reduced regardless of their qualitative diversity.

A problem both theoretical and practical is how one would be to determine the quantity of information, respectively, that of least choice as to real historical events. In general, it depends on the relative probability of a certain choice to the most probable one. The closer is the probability of the choice at issue to the maximal one in the given state of value field, the less is the choice, i.e. the closer is it to the state of maximal probability or "least choice".

To be utilizable practically, one needs: (1) the fan of all probable choices and decisions available in a certain historical problem; (2) their probability according the correspondence to the then or current value field; (3) the exclusion of the most probable ones among them due to the changed or forthcoming to change external circumstances. At last (4), the determination of those, relevant to the new circumstances, which are most probable according to the current value field. One should mean furthermore the difference between the estimation of the researchers historians post factum from that of the real actors, ex-ante. So, the latter is crucial for the relevance of made decision, respectively choice.

One can illustrate the described algorithm by the discussed already investigation of Fogel about the railroads. The quantity of social saving of the development of railroads to the counterfactual pathway to be developed the existing then transport demonstrates that (1) there have exist external obstacles for the latter or that (2) the innovation of railroads corresponds better to the then values therefore conditioning to be preferred, or (3) any combination of both.

The examples is directed, first of all, to make clear that the law of least choice combines the subjective historical causality in (2) with the objective one in (1) in a single and inseparable whole of historical choice.

A fundamental methodological idea of mathematical history consists in that unification of "object" and "subject" in history, borrowed from quantum mechanics: one of the greatest contribution to the epistemology of classical science where they are disjunctively separated from each other resulting as the "transparency" of the apparatus as on the fundamental distinguishability of objective probability from subjective probability. Quantum mechanics was forced to reject both, furthermore embedded in its formalism lent to mathematical history in the present research.

Thus, the corresponding merging of subject and object in history is intended by mathematical history due to the utilization of the same mathematical structure; and vice versa: the eventual necessity to be merged the epistemological subject and object in history justifies the mathematical history able to do it.

"Subject" and "object" in history, and then explicated in mathematical history, are merged in the concept of choice therefore postulating to be fundamental. There exists choice, there is choice, which can be embodied as in a human decision as in its limitation by external circumstances as well as any combination of the former and the latter. The mathematization of history implies and follows from their indistinguishability. That property of mathematical history can be understood also as the initial sense and motivation to be established.

So, the interpretation of the law of least choice means that subjective and objective choice cannot be separated or distinguished from each other in the "least choice" . 


\section{TO THE COEXISTENCE OF FREE WILL AND NECESSITY IN MATHEMATICAL HISTORY}

The crucial dependence on free will in history, being furthermore a humanitarian science, is obvious. There exist two fundamental ways for it to be available in history: in the decisions of actors as well as in the interpretations of historical discourse. Hermeneutics especially Gadamer's justifies its foundation by their unification. Mathematical history accepts it as well, however linking it to physical and mathematical transcendentalism rather than only to philosophical and historical transcendentalism as the original hermeneutics might be interpreted to do.

On the other hand, physics (before quantum mechanics) and mathematics as well as all the classic, rigorous, experimental or empiric science is based on the postulate of the necessity of scientific truths, only discovered by humans later or earlier. The opposition of free will and necessity is unified with another fundamental modern opposition of human being to nature identifying both former members and both latter members separately and then opposing them to each other as human free will to the necessity of nature: furthermore, the last opposition is relevant to the classical philosophical transcendentalism.

History as an objective science tries to distinguish disjunctively the area of free will limited only ex-ante and only to the historical actors from that of necessity kept only post factum and only to historians by means of the concept of historical fact granting it as an objective fact by itself and regardless of any interpretation, discourse or narrative added only externally and accidentally to it, and therefore, similar enough to any fact of classical science allowing for truth to be understood as adequacy.

However, philosophical and historical hermeneutics rejects that postulate of historical fact revising it to be as interpretable in many ways as merged with its interpretation inseparably. Rather paradoxically, but only at first glance, that merging is relevant to the establishment of mathematical history by involving the mathematical formalism of quantum mechanics as this is demonstrated in the paper.

There exists theorems in quantum mechanics (Conway, Kochen 2006; 2008) called by their authors "free will theorems" inferring them from a few mathematical statements valid in quantum mechanics and special relativity. The philosophical interpretation of them coined by the authors themselves consists in the following conclusion. If the enumerated premises hold, and the experimenter (i.e. human being) in the area of quantum mechanics possesses free will, the investigated quantum entity (such as an electron, for example) has to possess free will in the same rigorous, physical and mathematical meaning as the experimenter ${ }^{26}$.

In fact, that solution of the philosophical problem of the relation or coexistence of free will and necessity, implied by the mathematical structure of quantum mechanics and special relativity, is fundamentally different from that underlying the epistemological and ontological foundations of classical mechanics. Unlike the latter, it distinguishes two different oppositions, free will versus necessity, on the one hand, and human being versus necessity, on the other hand, allowing for any quantum entity to possess free will in the same meaning as a human being.

${ }^{26}$ Without being proved formally and mathematically, that shocking corollary from quantum mechanics has been known since a long time ago, also being in the base of Einstein's non-acceptance of quantum mechanics (Einstein 1926@) 
The modus tollens of the same interpretation would be: if the investigated quantum entity does not possess free will (as classical science would agree), the experimenter (i.e. a human being) does not possess free will as well (which classical science would reject). Those theorems demonstrate that the solutions of quantum mechanics and classical science are diametrically opposed to each other in a sense elucidated above.

Sharing the same formalism with quantum mechanics, mathematical history accepts the relevant premises for the free will theorems, and originating from it. The validity of those originating from special relativity can be justified as follows:

History takes place in the physical world therefore obeying its laws, particularly those of special relativity, and its formalism is to be interpretable after mathematical history. Thus, the premises originating from both quantum mechanics and special relativity can be related not less to mathematical history, and the validity of the free will theorems be reaffirmed in its scope as well.

The sense of the free will theorems as to mathematical history would be: the free will of the actors in history (i.e. human free will) implies the existence of "choice at all", "subjectless choice", (i.e. choice of the being or the universe). In other words, if the "subject" in a philosophical sense is able to choose, the relevant "object" is able not less, and thus, all in the totality and the totality itself. Then, the converse statement built by means of modus tollens holds as well:

If the "object" obeys necessity, particularly natural necessity, the subject, particularly human being, also does, and all in the totality as well as the totality itself obeys necessity or natural necessity. Both viewpoints to the relation of free will (freedom) and necessity are admissible equally, but this does not imply any contradiction (unlike classical science) as they are dual or complementary to each other in the sense of quantum mechanics suggested by Niels Bohr.

That kind of solution of the fundamental philosophical problem (at least as to the Western classical philosophy) about the relation of free will (freedom) and necessity) originates still from the quantum neo-Pythagoreanism postulating for the base of the world or the being to be mathematical and embodied in Hilbert arithmetic and in the qubit Hilbert space, and borrowed by mathematical history:

Hilbert arithmetic as a triple of Peano arithmetics or respectively bits of classical information represent the one dual aspect of the opposition "free will (freedom) - necessity", namely the latter: necessity; and accordingly, the other dual aspect (that of the qubit Hilbert space" means free will and freedom. However, neither of both aspects can be assigned unambiguously to "subject" (respectively, "object") once the totality has been involved for it merges them definitively: for example, choice is both subjective and objective in mathematical history and quantum mechanics since the totality is a necessary condition (though often, implicit) for each of them to be established and embedded in the mathematical formalism shared by both.

The relation of free well (freedom) and necessity in history can be visualized even only by the concept of hermeneutical circle ${ }^{27}$. The circle seen "inside" suggests many alternatives of both historical decisions ex-ante and their interpretations post factum. That external

${ }^{27}$ It satisfies the fundamental, formal and logical tautology as this was demonstrated in the text already. 
viewpoint means the involvement of actors and interpreters in the single historical fact as an "atom" of history as it is, seen "outside", without "empathy" to the taken place, but "objectively". The former corresponds to the one dual aspect, that of free will (freedom); and the latter, to the other one, that of necessity.

Both aspects can be merged only in the totality therefore involving historical and mathematical transcendentalism necessary for mathematical history or serving as its justification in the discourse of foundation.

XVII INSTEAD OF CONCLUSION: WHAT MATHEMATICAL HISTORY STUDIES

Mathematical history can be seen in three dissimilar ways from three fundamentally different viewpoints: (1) as a Pythagorean philosophy of history from the viewpoint of historical and mathematical transcendentalism; (2) as a theoretical history establishing general laws such as that of least choice from the viewpoint of the mathematical formalism borrowed from quantum mechanics; and (3), as a methodology for historical research after the interpretation of that mathematical formalism in properly historical and/ or social terms. So, mathematical history unifies the study in those three directions, which will be considered in detail:

1. The fundamental ontology, however interpreted to be neo-numerical (in fact, substituting the Pythagorean sacral "number" by the contemporary profane "mathematical structure") by itself, implies for the "regional ontology" of history to be the same and thus numerical as well. Mathematical history originates from that historical ontology, and the way in which this is possible is researched by relevant, i.e. neo-Pythagorean philosophy of history.

A "peculiarity" featuring the relation of the fundamental ontology and historical ontology is inherited in mathematical history as a philosophy of history from all philosophical doctrines originating from transcendentalism or relevant to the conception of the totality. This is the aspect of identity or duality of the fundamental ontology and the regional historical ontology ${ }^{28}$, exemplifiable by the identity of philosophical transcendentalism with historical and mathematical transcendentalism ${ }^{29}$ as in the interpretation of historical dialectics or historical hermeneutics described above.

The cyclic structure of the totality meant by the fundamental, formal and logical tautology (e.g. after physical and mathematical transcendentalism) can be interpreted as embodying the wholeness (or the "totality") of history into any historical decision by its actors or any

\footnotetext{
${ }^{28}$ Probably, Hegel was the first great philosopher who demonstrated convincingly that his dialectics (originating from transcendentalism, in fact) can be interpreted as history therefore able to unify history of philosophy and philosophy of history.

${ }^{29}$ Historical and mathematical transcendentalism is meant only here as a doctrine of philosophy and history, thus unfalsifiable: on the contrary, the same concept, "historical and mathematical transcendentalism" designated a scientific hypothesis, and thus, falsifiable by rejecting facts or by formal and logical inconsistency. The single, but very essential difference between the two uses of "historical ad mathematical transcendentalism consists only in adding the consistent and independent property of falsifiability, respectively of non-falsifiability. The latter is a crucial disadvantage for any scientific theory, but a necessary condition for many metaphysical theories interpreted usually as philosophical. The transition between them can be accomplished, for example, by the interpretation of the fundamental, formal and logical tautology as a proper logical tautology, thus true identically (or "analytically" after transcendentalism) or as a definitive property of the totality meant in the "regional ontology" of mathematical history (or "synthetically") as far as the totality admits synthetic statements referring to it a priori.
} 
interpretation of history by its researches. Any of both involves a choice, and in virtue of this, information.

2. Mathematical history underlain by a very well elaborated mathematical formalism withal exceptionally successfully utilized by quantum mechanics allows for rigorous and thus testable laws of history, however absolutely inaccessible as to the usual historical research being fundamentally descriptive.

An example is the law of least choice inferable from the conservation of quantum information and discussed in the paper.

Furthermore, exact mathematical equivalents of unobservable and hypothetical histories such as counterfactual histories can be suggested as observed elements or collection in the real history: such as states of value field.

3. An absolutely certain methodology for historical research (especially as to counterfactual ones) is deducible from mathematical history. Its main objectivities are to reveal the methods by which one can suggest mathematical equivalents (such as "wave functions" or states of "value fields") of historical processes in different cultures and civilizations or to resolve the converse problem: which the interpretations of the elements of certain wave functions would be in terms of the usual historical discourse.

So, mathematical history unifies and studies the relevant philosophy of history, of Pythagorean kind, mathematical models of theoretical history, and methodology of historical research.

However, the present research, the subject of which is the foundations and justifications of mathematical of mathematical history first of all, is concentrated only to the first scope from the enumerated subareas though the examples in it can be related to the others. 


\section{References:}

Conway, John; Kochen, Simon (2006) "The Free Will Theorem," Foundations of Physics 36 (10): 1441-1473.

Conway, John; Kochen, Simon (2009) "The Strong Free Will Theorem," Notices of the AMS 56 (2): 226-232.

Einstein, Albert (1926) "Letter to Max Born, 16 December 1926," in: Albert Einstein Max Born Briefwechsel 1916 - 1955 (kommentiert von Max Born), München, Nymphenburger Verlagshandlung, 1969, pp. 129-130.

Fogel, Robert (1964) Railroads and American Economic Growth: Essays in Econometric History, Baltimore, The Johns Hopkins Press.

Fogel, Robert; Engerman, Stanley (1974) Time on the Cross: The Economics of American Negro Slavery, Boston; Little, Brown and Co.

Heidegger, Martin (1947) Über den Humanismus, Frankfurt AM, Vittorio Klostermann.

Kochen, Simon; Specker, Ernst (1968) "The problem of hidden variables in quantum mechanics," Journal of Mathematics and Mechanics 17 (1): 59-87.

Neumann, John von (1932) Mathematische Grundlagen der Quantenmechanik, Berlin, Springer.

Penchev, Vasil (2020 October 20) "Two deductions: (1) from the totality to quantum information conservation; (2) from the latter to dark matter and dark energy," https://ssrn.com/abstract=3683658

Penchev, Vasil (2020 October 19) "The case The Case of Quantum Mechanics Mathematizing Reality: The 'Superposition' of Mathematically Modeled and Mathematical Reality: Is There Any Room for Gravity?" https://ssrn.com/abstract=3683342

Penchev, Vasil (2020 October 5) "Quantum-Information Conservation. The Problem About 'Hidden Variables', or the 'Conservation of Energy Conservation' in Quantum Mechanics: A Historical Lesson for Future Discoveries," https://ssrn.com/abstract=3675319

Penchev, Vasil (2020 August 25) "The Relationship of Arithmetic as Two Twin Peano Arithmetic(s) and Set Theory: A New Glance from the Theory of Information," https://ssrn.com/abstract $=3656179$

Penchev, Vasil (2020 July 10) "Quantum Information as the Information of Infinite Series," https://ssrn.com/abstract $=3630063$ 